\title{
STABLE MAPS TO LOOIJENGA PAIRS: ORBIFOLD EXAMPLES
}

\author{
PIERRICK BOUSSEAU, ANDREA BRINI, AND MICHEL VAN GARREL \\ In grateful memory of Boris Anatol'evich Dubrovin, 1950-2019
}

\begin{abstract}
In 15] we established a series of correspondences relating five enumerative theories of log Calabi-Yau surfaces, i.e. pairs $(Y, D)$ with $Y$ a smooth projective complex surface and $D=$ $D_{1}+\cdots+D_{l}$ an anticanonical divisor on $Y$ with each $D_{i}$ smooth and nef. In this paper we explore the generalisation to $Y$ being a smooth Deligne-Mumford stack with projective coarse moduli space of dimension 2, and $D_{i}$ nef $\mathbb{Q}$-Cartier divisors. We consider in particular three infinite families of orbifold log Calabi-Yau surfaces, and for each of them we provide closed form solutions of the maximal contact log Gromov-Witten theory of the pair $(Y, D)$, the local Gromov-Witten theory of the total space of $\bigoplus_{i} \mathcal{O}_{Y}\left(-D_{i}\right)$, and the open Gromov-Witten of toric orbi-branes in a CalabiYau 3-orbifold associated to $(Y, D)$. We also consider new examples of BPS integral structures underlying these invariants, and relate them to the Donaldson-Thomas theory of a symmetric quiver specified by $(Y, D)$, and to a class of open/closed BPS invariants.
\end{abstract}

\section{CONTENTS}

1. Introduction 1

2. Setup and main results

3. Example I 14

4. Example II 18

5. Example III

6. BPS invariants

References

\section{INTRODUCTION}

In [15], we established a series of correspondences between a priori distinct enumerative theories of Gromov-Witten (GW)/Donaldson-Thomas (DT) type associated to smooth log Calabi-Yau surface of maximal boundary with nef boundary components, or nef Looijenga pairs: these are pairs $(Y, D)$ where $Y$ is a smooth projective surface and $\left|-K_{Y}\right| \ni D=D_{1}+\cdots+D_{l}$ is an anticanonical normal crossings divisor with $l>1$ smooth and nef irreducible components $D_{j}$. For a nef Looijenga pair we

This project has been supported by the European Union's Horizon 2020 research and innovation programme under the Marie Sklodowska-Curie grant agreement No 746554 (M. vG.), the Engineering and Physical Sciences Research Council under grant agreement ref. EP/S003657/2 (A. B.) and by Dr. Max Rössler, the Walter Haefner Foundation and the ETH Zürich Foundation (P. B. and M. vG.). 
proved an equivalence between the log GW theory of the pair $(Y, D)$, the local GW theory of the total space of the sum of dual line bundles to the irreducible components $D_{j}$, the open GW theory of Aganagic-Vafa branes in a Calabi-Yau threefold associated to $(Y, D)$, the DT theory of a symmetric quiver specified by $(Y, D)$, and a variety of BPS invariants considered by Klemm-Pandharipande, Ionel-Parker, and Labastida-Mariño-Ooguri-Vafa. Moreover, we provided closed-form solutions for the calculation of the these invariants in all the finitely many deformation families of such pairs.

In this companion note we explore the extension of such correspondences to include orbifolds, and provide compelling evidence that the bulk of the correspondences put forward in [15] generalise to this setting essentially verbatim. We consider pairs $\left(\mathcal{Y}, D=D_{1}+\cdots+D_{l}\right)$ where $\mathcal{Y}$ is a smooth complex Deligne-Mumford stack with coarse moduli space a normal Gorenstein projective surface $Y,(Y, D)$ is log smooth (in particular, the singularities are concentrated along the codimension 2 strata of $D), D \in\left|-K_{Y}\right|$, and the irreducible components $D_{j}$ are nef and $\mathbb{Q}$-Cartier for all $j=1, \ldots, l$. In particular we will exemplify how and to what extent our circle of correspondences in 15 generalises to this context in three infinite families of log Calabi-Yau orbifolds:

Example I: in this example, $Y$ is taken to be the weighted projective plane $\mathbb{P}(1, a, b)$ for $a, b$ positive coprime integers with fan given in Figure 1.1. This surface has two orbifold singularities that are locally quotients of $\mathbb{C}^{2}$ by the finite cyclic group $\mu_{a}$, resp. $\mu_{b}$, and there is a toric line $D_{(b, a)}$ that joins both. Extending $D_{(b, a)}$ to an anticanonical divisor by adding a general member $D_{2}$ of $\left|-K_{Y}-D_{(b, a)}\right|$ gives the Looijenga orbi-pair $\mathbb{P}(1, a, b):=\left(\mathbb{P}(1, a, b), D=D_{(b, a)}+D_{2}\right)$. It is non-toric since the topological Euler characteristic of the complement of $D$ is $\chi(\mathbb{P}(1, a, b) \backslash D)=1$.

Example II: in this case we blow up $\mathbb{P}(1, a, b)$ in a smooth point of $D_{2}$. We denote the resulting surface with its choice of 2-component boundary by $Y_{(a, b)}^{[2]}$.

Example III: blowing up $\mathbb{P}(1, a, b)$ in a smooth point of one of its toric divisors leads to a non-toric nef orbi-Looijenga pair with $l=3$, which we denote by $Y_{(a, b)}^{[3]}$.

\section{SETUP AND MAIN RESULTS}

2.1. The enumerative theories. Let $(Y, D)$ be a log smooth log Calabi-Yau surface with $D=$ $D_{1}+\cdots+D_{l}$ and each $D_{j}$ irreducible and let $d \in \mathrm{H}_{2}(Y, \mathbb{Z})$. Provided that étale-locally around each singularity $Y(D)$ is isomorphic to a toric variety with its toric boundary, $Y(D)$ is $\log$ smooth. In particular this applies to Examples I-III. We will use the short-hand notation $Y(D)$ to denote the log-scheme obtained by taking the divisorial $\log$ structure induced by $D$ on $Y$. For $n \geq 0$, denote by $[n]_{q}$ the $q$-number $q^{\frac{n}{2}}-q^{-\frac{n}{2}}$, as well as the symmetrised $q$-factorials $[n]_{q} !:=\prod_{i=1}^{n}[i]_{q}$ and $q$-binomials $\left[\begin{array}{c}n \\ m\end{array}\right]_{q}:=[n]_{q} ! /\left([m]_{q} ![n-m]_{q} !\right)$.

2.1.1. All genus $\log G W$ invariants. Let $g \geq 0$. We are (virtually) counting genus $g$ degree $d$ curves in $X$ that have prescribed tangency conditions along the boundary $D$, namely we require the curves to meet each of $D_{j}$ in one point of maximal tangency $d \cdot D_{j}$. This is a moduli problem of virtual dimension $g+l-1$. 


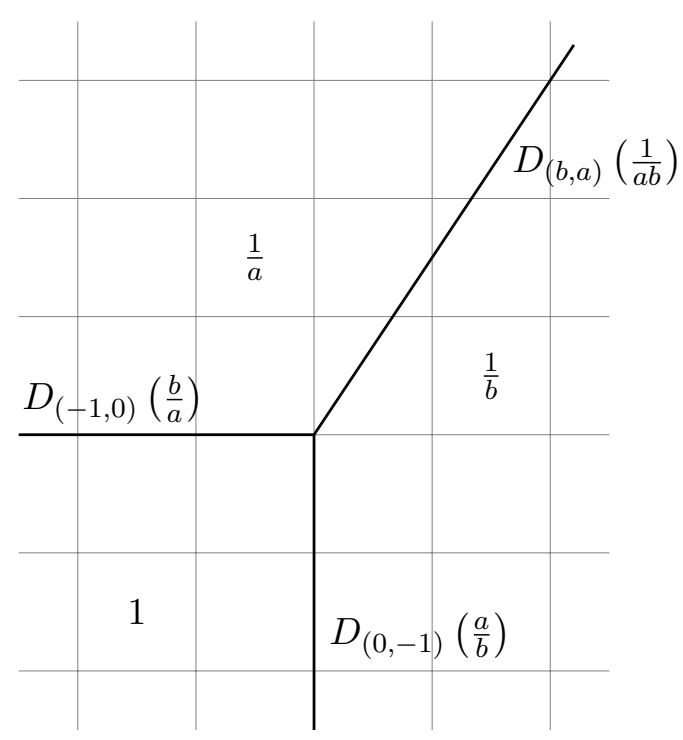

Figure 1.1. The fan of $\mathbb{P}(1, a, b)$ with toric intersection numbers

Log smoothness of $Y(D)$ guarantees the existence of the corresponding moduli space of basic stable log maps $\overline{\mathrm{M}}_{g, m}^{\log }(Y(D), d)$ as constructed by Abramovich-Chen [1,21 and Gross-Siebert 44 by encoding the tangency conditions via log structures. It admits a rank $g$ vector bundle $\mathbb{E}$ whose fiber over $f: C \rightarrow Y(D)$ is the vector space $\mathrm{H}^{0}\left(C, \omega_{C}\right)$ of sections of the dualising sheaf of the domain curve. To cut down the virtual dimension to 0 , we require the curves to pass through $l-1$ general points in the interior $Y \backslash D$ and further cap the virtual fundamental class by the top Chern class $\lambda_{g}:=c_{g}(\mathbb{E})$ of $\mathbb{E}$, leading to invariants

$$
N_{g, d}^{\log }(Y(D)):=\int_{\left[\overline{\mathrm{M}}_{g, l-1}^{\log }(Y(D), d)\right]^{\mathrm{vir}}}(-1)^{g} \lambda_{g} \prod_{j=1}^{l-1} \mathrm{ev}_{j}^{*}([\mathrm{pt}]),
$$

where $\mathrm{ev}_{j}: \overline{\mathrm{M}}_{g, l-1}^{\log }(Y(D), d) \rightarrow X$ is the morphism given by evaluation at the $j$ th point. We denote by $N_{d}^{\log }(Y(D)):=N_{0, d}^{\log }(Y(D))$.

We package the invariants into the fixed-degree, all-genus generating function

$$
\mathrm{N}_{d}^{\log }(Y(D))(\hbar):=\frac{1}{\left(2 \sin \left(\frac{\hbar}{2}\right)\right)^{l-2}} \sum_{g \geqslant 0} N_{g, d}^{\log } \hbar^{2 g-2+l} .
$$

By a combination of [11, 13] (see [15, Proposition 4.2]), after the change of variable $q=e^{i \hbar}$, $\mathrm{N}_{d}^{\log }(Y(D))(\hbar)$ is the power series expansion in $\hbar$ of polynomials $\mathbf{N}_{d}^{\log }(Y(D))(q)$ in $q^{\frac{1}{2}}$.

The invariants $N_{g, d}^{\log }(Y(D))$ can be naturally viewed as invariants of the 3 -fold $Y(D) \times \mathbb{A}^{1}$. Indeed, the Gromov-Witten obstruction theories for stable maps to the surface $Y(D)$ and the 3 -fold $Y(D) \times$ $\mathbb{A}^{1}$ differ by the space $H^{1}\left(C, \mathcal{O}_{C}\right)\left[54\right.$, Lemma 7], which by Serre duality is dual to $H^{0}\left(C, \omega_{C}\right)$. Thus, the insertion of $(-1)^{g} \lambda_{g}$ in the Gromov-Witten theory of the surface $Y(D)$ exactly reproduces Gromov-Witten invariants of the 3 -fold $Y(D) \times \mathbb{A}^{1}$. Essentially for the same reason, the class $(-1)^{g} \lambda_{g}$ appears also naturally in the higher genus extension of the log-local correspondence for a smooth divisor presented in [16]. In [15], in particular Section 1.4, we explained how higher genus 
$\log$ Gromov-Witten invariants of log Calabi-Yau surfaces with insertion of $(-1)^{g} \lambda_{g}$ fit into a web of log-local-open correspondences. In the present paper, we use the invariants $N_{g, d}^{\log }(Y(D))$ to extend this web of correspondences to the orbifold setting.

2.1.2. Local $G W$ invariants. A different class of invariants of $(Y, D)$ arises by considering the local theory of $\operatorname{Tot}\left(\bigoplus_{j} \mathcal{O}_{Y}\left(-D_{j}\right)\right)$. This is a non-compact Calabi-Yau- $(l+2)$ fold, and since for $l>1$ there are no non-zero Gromov-Witten invariants with point insertions for $g>0$, we restrict below to genus 0 .

Suppose $Y$ admits a presentation as a Gorenstein GIT quotient $Y=Z / / G$ for a complex smooth projective variety $Z$ and reductive group $G$, and write $\mathcal{Y}=[Z / / G]$ for the Deligne-Mumford quotient stack it represents. While the Gorenstein surface $Y$ (with trivial log structure) is not smooth, $Y(D)$ and $\mathcal{Y}$ are smooth in the respective categories by definition. The genus 0 local GW theory of $Y(D)$ is a (virtual) count of rational orbi-curves in the $(l+2)$-dimensional non-compact Calabi-Yau orbifold $\mathcal{E}_{Y(D)}:=\operatorname{Tot}\left(\oplus_{i=1}^{l}\left(\mathcal{O}_{\mathcal{Y}}\left(-D_{i}\right)\right)\right)$ with coarse space $E_{Y(D)}:=\operatorname{Tot}\left(\oplus_{i=1}^{l}\left(\mathcal{O}_{Y}\left(-D_{i}\right)\right)\right)$. Given that the $\log \mathrm{GW}$ theory is insensitive to the twisted sector, we will only be interested in the untwisted sector of the orbifold GW theory of $\mathcal{E}_{Y(D)}[2]$.

Denote by $\overline{\mathrm{M}}_{0, m}(\mathcal{Y}, d)$ the moduli stack of twisted genus $0 \mathrm{~m}$-marked stable maps $[f: \mathcal{C} \rightarrow \mathcal{Y}]$ with $f_{*}([\mathcal{C}])=d$ and $\mathcal{C}$ an $m$-pointed twisted curve. We write $\overline{\mathrm{M}}_{0, m}(Y, d)$ for the substack of twisted stable maps such that the image of the evaluation maps is contained in the age zero component of the inertia stack of $\mathcal{Y}$. The moduli stack $\overline{\mathrm{M}}_{0, m}(\mathcal{Y}, d)$ has a perfect obstruction theory, inducing a virtual fundamental class

$$
\left[\overline{\mathrm{M}}_{0, m}(Y, d)\right]^{\mathrm{vir}} \in \mathrm{H}_{2 \mathrm{vdim}}\left(\overline{\mathrm{M}}_{0, m}(Y, d), \mathbb{Q}\right),
$$

where $\operatorname{vdim}=-K_{Y} \cdot d+m-1$.

Assuming that $d \cdot D_{j}>0$, there is a rank $-K_{Y} \cdot d-l$ obstruction vector bundle $\mathrm{Ob}_{D}$ on $\overline{\mathrm{M}}_{0, m}^{\log }(Y, d)$ with fibre $\mathrm{H}^{1}\left(\mathcal{C}, f^{*} \bigoplus_{j=1}^{l} \mathcal{O}_{\mathcal{Y}}\left(-D_{j}\right)\right)$ over a twisted stable map $[f: \mathcal{C} \rightarrow \mathcal{Y}]$. It is defined as $\mathrm{Ob}_{D}:=R^{1} \pi_{*}\left(f^{\text {univ }}\right)^{*}\left(\bigoplus_{j=1}^{l} \mathcal{O}_{Y}\left(-D_{j}\right)\right)$ for $\pi: \mathcal{C}^{\text {univ }} \rightarrow \overline{\mathrm{M}}_{0, m}(\mathcal{Y}, d)$ the universal curve and $f^{\text {univ }}$ : $\mathcal{C}^{\text {univ }} \rightarrow \mathcal{Y}$ the universal twisted stable map. Restricting to the component of the inertia stack of age zero, we obtain the virtual fundamental class

$$
\left[\overline{\mathrm{M}}_{0, m}\left(E_{Y(D)}, d\right)\right]^{\mathrm{vir}}:=\left[\overline{\mathrm{M}}_{0, m}(Y, d)\right]^{\mathrm{vir}} \cap c_{\mathrm{top}}\left(\mathrm{Ob}_{D}\right) \in \mathrm{H}_{2(l-1+m)}\left(\overline{\mathrm{M}}_{0, m}(Y, d), \mathbb{Q}\right) .
$$

Restricting to the untwisted sector yields evaluations maps $\mathrm{ev}_{j}: \overline{\mathrm{M}}_{0, m}(Y, d) \rightarrow Y$ and we define the following two classes of local invariants

$$
\begin{aligned}
N_{d}^{\mathrm{loc}}(Y(D)) & :=\int_{\left[\overline{\mathrm{M}}_{0, l-1}\left(E_{Y(D)}, d\right)\right]^{\mathrm{vir}}} \prod_{j=1}^{l-1} \mathrm{ev}_{1}^{*}([\mathrm{pt}]), \\
N_{d}^{\mathrm{loc}, \psi}(Y(D)) & :=\int_{\left[\overline{\mathrm{M}}_{0,1}\left(E_{Y(D)}, d\right)\right]^{\mathrm{vir}}} \operatorname{ev}_{1}^{*}([\mathrm{pt}]) \psi_{1}^{l-2},
\end{aligned}
$$

where $\psi_{i}=c_{1}\left(\mathbb{L}_{i}\right)$ is the first Chern class of the $i^{\text {th }}$ tautological line bundle on $\overline{\mathrm{M}}_{0, m}(Y, d)$. 
2.1.3. All genus open $G W$ invariants. In [15, Construction 6.4], we showed how to associate to a smooth Looijenga pair $Y(D)$ satisfying certain positivity properties [15, Definition 6.3] a triple $Y^{\mathrm{op}}(D)=(X, L, \mathrm{f})$ with $X$ a semi-projective toric Calabi-Yau 3 -fold, $L=L_{1} \cup \cdots \cup L_{l-1}$ a disjoint union of $l-1$ Aganagic-Vafa toric Lagrangians $[7]$ in $X$ and $f$ a framing for $L$. At first approximation, $X$ is the total space of $K_{Y \backslash\left(D_{1} \cup \ldots \cup D_{l-1}\right)}$, the $L_{j} \simeq S^{1} \times \mathbb{R}^{2}$ are Lagrangians that contract to a vanishing cycle $\left[S^{1}\right]$ of $Y$ near $D_{j}$, and $\mathrm{f}$ is determined by the compactification given by adding back the $D_{j}, j<l$. See 15. Construction 6.4], the framing determines the compactification of (a toric limit of) $Y \backslash\left(D_{1} \cup \cdots \cup D_{l-1}\right)$ to (a toric limit of) $Y$. At the level of their associated polyhedra, the framing determines the additional halfspaces to intersect with to go from from the polyhedron of $Y \backslash\left(D_{1} \cup \cdots \cup D_{l-1}\right)$ to the polytope of $Y$ (with anticanonical polarizations). The framings correspond to the slopes of the edges.

It is immediate to verify from [15, Construction 6.4] that the above generalises to the case of Looijenga orbi-pairs $Y(D)$, for which $Y^{\mathrm{op}}(D)=(X, L, \mathrm{f})$ is in general a semi-projective Gorenstein orbifold $X$ with fractionally framed orbifold toric Lagrangians $(L, \mathrm{f})$ [17. The orbifold case introduces a small modification. If $Y$ has orbifold singularities at the toric 0 -strata, we obtain rational framings. More precisely, denote by $\mathbb{A}^{1}$ one of the toric strata corresponding to an outer edge of the toric graph with framing. Then the $\mathbb{A}^{1}$ is compactified in $Y$ by adding a point in $Y$ that is a cyclic quotient singularity with isotropy group $\mathbb{Z} / r \mathbb{Z}$, for $r$ the denominator of the framing. Adapting it to the orbifold case, the construction moreover induces a natural injection $\iota: \mathrm{H}_{2}^{\mathrm{rel}}\left(Y^{\mathrm{op}}(D), L ; \mathbb{Z}\right) \hookrightarrow \mathrm{A}_{1}(Y, \mathbb{Z})$ as we review in Section 2.2.2, with all the curve classes $d \in \mathrm{H}_{2}(Y, \mathbb{Z})$ lying in its image. In Examples I-III, $X$ and $L$ will always be smooth, but the framing $\mathrm{f}$ will be fractionally shifted by rational numbers $f_{i}=p_{i} / r_{i}$ from the canonical framing on each connected component $L_{i}$ of $L$.

The open GW theory of $Y^{\mathrm{op}}(D)$ was defined in the algebraic category ${ }^{1}$ in 50 . Given partitions $\mu_{i}$ of length $\ell\left(\mu_{i}\right), i=1, \ldots, l-1$, there is a virtual dimension zero moduli space $\overline{\mathrm{M}}_{g ; \beta ; \mu_{1}, \ldots, \mu_{l-1}}\left(Y^{\mathrm{op}}(D)\right)$ of relative degree $\beta$ open stable morphisms to $Y^{\text {op }}$ from genus- $g$, open Riemann surfaces with $\sum_{i=1}^{l-1} \ell\left(\mu_{i}\right)$ connected components of the boundary mapping to $L_{i}$ with winding numbers around $S^{1} \hookrightarrow L_{i}$ equal to the parts of $\mu_{i}$. The corresponding open GW invariants,

$$
O_{g, \beta, \vec{\mu}}\left(Y^{\mathrm{op}}(D)\right)=\int_{\left[\overline{\mathrm{M}}_{g ; \beta ; \vec{\mu}}\left(Y^{\mathrm{op}}(D)\right)\right]^{\mathrm{vir}}} 1
$$

can be encoded into formal generating functions

$$
\mathrm{O}_{\beta ; \vec{\mu}}\left(Y^{\mathrm{op}}(D)\right)(\hbar):=\sum_{g} \hbar^{2 g-2+\ell(\vec{\mu})} O_{g ; \beta ; \vec{\mu}}\left(Y^{\mathrm{op}}(D)\right)
$$

with $\ell(\vec{\mu})=\sum_{i=1}^{s} \ell\left(\mu_{i}\right)$. We will write simply $O_{g ; \beta}\left(Y^{\mathrm{op}}(D)\right)$ and $\mathrm{O}_{\beta}\left(Y^{\mathrm{op}}(D)\right)(\hbar)$ for the $(l-1)$ holed open GW invariants obtained when $\mu_{i}=\left(m_{i}\right)$, which are then determined by the class $\beta \in \mathrm{H}_{2}^{\mathrm{rel}}\left(Y^{\mathrm{op}}(D), L ; \mathbb{Z}\right)$.

\footnotetext{
${ }^{1}$ See 17 for a definition of open GW invariants of toric orbifold Lagrangians using localisation, and 35 for a definition for smooth toric Lagrangians with fractional framing using relative GW theory.
} 
2.1.4. Quiver DT invariants. Let $\mathrm{Q}$ be a symmetric quiver with $n$ vertices and, for dimension vectors $\mathrm{d}=\sum_{i} d_{i} v_{i}, \mathrm{e}=\sum_{i} e_{i} v_{i} \in \mathbb{N Q}_{0}=\mathbb{N} v_{1}+\cdots+\mathbb{N} v_{n}$, denote by $E_{\mathrm{Q}}(\mathrm{d}, \mathrm{e})$ the Gram matrix of the Euler form

$$
E_{\mathrm{Q}}(\mathrm{d}, \mathrm{e}):=\sum_{i=1}^{n} d_{i} e_{i}-\sum_{\alpha: v_{i} \rightarrow v_{j}} d_{i} e_{j}
$$

The motivic DT invariants $\mathrm{DT}_{\mathrm{d} ; i}(\mathrm{Q})$ of $\mathrm{Q}$ are defined from the plethystic generating function

$$
\operatorname{Exp}\left(\frac{1}{[1]_{q}} \sum_{\mathrm{d} \neq 0} \sum_{i \in \mathbb{Z}} \operatorname{DT}_{\mathrm{d} ; i}(\mathrm{Q}) \mathrm{x}^{\mathrm{d}}\left(-q^{1 / 2}\right)^{-i}\right)=\sum_{\mathrm{d} \in \mathbb{N}^{n}} \frac{\left(-q^{1 / 2}\right)^{E_{Q}(\mathrm{~d}, \mathrm{~d})} \mathrm{x}^{\mathrm{d}}}{\prod_{i=1}^{n}(q ; q)_{d_{i}}}
$$

where $\mathrm{x}^{\mathrm{d}}=\prod_{i=1}^{n} x_{i}^{d_{i}}$. Using the terminology of $[10, \S 3.3]$, the right-hand side is the generating series of Poincaré rational functions of the stacks of representations of $Q$. The numerical DT invariants $\mathrm{DT}_{d}^{\text {num }}(\mathrm{Q})$ are non-negative 32$]$ integers defined by

$$
\operatorname{DT}_{\mathrm{d}}^{\text {num }}(\mathrm{Q}):=\sum_{i \in \mathbb{Z}}(-1)^{i} \mathrm{DT}_{\mathrm{d}, i}(\mathrm{Q})
$$

2.1.5. BPS invariants. For a Looijenga orbi-pair $Y(D)$, we define open BPS numbers as in 15 , Equation (1.21)] by

$$
\Omega_{d}(Y(D))(q):=[1]_{q}^{2}\left(\prod_{i=1}^{l} \frac{1}{\left[d \cdot D_{i}\right]_{q}}\right) \sum_{k \mid d} \frac{(-1)^{d / k \cdot D+l} \mu(k)}{[k]_{q}^{2-l} k^{2-l}} \mathrm{~N}_{d / k}^{\log }(Y(D))(-\mathrm{i} k \log q) .
$$

We will also denote just by $\Omega_{d}(Y(D))$ the genus-zero limit $\Omega_{d}(Y(D))(1)$.

The log-open correspondence of Theorem 2.2 below implies that, for Examples I-III,

$$
\Omega_{d}(Y(D))(q)=[1]_{q}^{2} \prod_{i=1}^{l-1} \frac{r_{i}\left(d \cdot D_{i}\right)}{\left[d \cdot D_{i}\right]_{q}} \sum_{k \mid d} \frac{\mu(k)(-1)^{\sum_{i=1}^{l-1} d / k \cdot D_{i}\left(r_{i}+1\right)}}{k} \mathrm{O}_{\iota^{-1}(d / k)}\left(Y^{\mathrm{op}}(D)\right)(-\mathrm{i} k \log q),
$$

where $f_{i}=p_{i} / r_{i}$ with $\left(p_{i}, r_{i}\right)=1$ is the framing of the $i^{\text {th }}$ orbifold Aganagic-Vafa Lagrangian in $Y^{\mathrm{op}}(D)$. Even though $\Omega_{d}(q)$ can at most be expected to be a rational function of $q^{1 / 2}$, heuristically, and for smooth, integrally framed $Y^{\mathrm{op}}(D)$ 48, 49, 52, 56], $\Omega_{d}(Y(D))(q)$ has an interpretation as generating function of BPS domain walls counts in a type IIA string compactification on $Y^{\mathrm{op}}(D)$, with its coefficient computing degeneracies of D2-branes with fixed spin and charge ending on a D4-brane wrapped around the Lagrangians $Y^{\mathrm{op}}(D)$. The formula $[2.12$ generalises 52 , Eq. 2.10] to the orbifold setting, with an additional factor keeping track of the fractional framing of the branes.

2.2. The correspondences. In our previous paper [15] we proposed that the invariants of the previous Section are related through a series of geometric correspondences. A conceptual explanation of these was provided in [15, Section 1.4], and we briefly recall it in Section 2.2.4. 
2.2.1. Numerical log-local. Our first result is the following

Theorem 2.1. Assume that $Y(D)$ is one of $\mathbb{P}(1, a, b), Y_{(a, b)}^{[2]}$ or $Y_{(a, b)}^{[3]}$. Then

$$
N_{d}^{\mathrm{loc}}(Y(D))=\left(\prod_{j=1}^{l} \frac{(-1)^{d \cdot D_{j}-1}}{d \cdot D_{j}}\right) N_{d}^{\log }(Y(D)) .
$$

Our proof of Theorem 2.1 follows from a stronger result, wherein we give a complete closed-form solution of both sides of $(2.13)$ in all degrees. In the case of an irreducible smooth nef divisor, the correspondence between genus $0 \log$ and local GW invariants was proven in all dimensions at the cycle-level in [36], with various extensions in $8,10,14,15,23,25,34,55,61,62$. The naive conjectural extension of this log-local correspondence at the cycle level for normal crossings divisors has been recently disproved [8,55]. However, the numerical version of the log-local correspondence for normal crossing divisors seems to hold in a number of cases of great interest: for example this was proved for point insertions of orbifold toric pairs in [14], and for point invariants of log CalabiYau surfaces with nef $D_{i}$ in [15]. Theorem 2.1 simultaneously provides a non-toric, orbifold version of the numerical version of the log-local correspondence of $14,36,2$

2.2.2. Log-open. Our second result is an orbifold generalisation of the higher genus log-open principle of [15, Conjecture 1.3]. Following [15, Definition 6.5], we canonically identify each curve degree $d \in \mathrm{H}_{2}(Y, \mathbb{Z})$ with a relative curve degree $\iota^{-1}(d)$ in $Y^{\mathrm{op}}(D)$. We recall how this identification works and adapt it to the orbifold setting. The class of a Riemann surface with boundary in $Y^{(\mathrm{op})}(D)$ is decomposed as an $l$-tuple $\left(\beta, \alpha_{1}, \ldots, \alpha_{l-1}\right)$. Here $\beta$ is a 2 -homology class, which decomposes as a sum of the homological 2-spheres corresponding to the compact toric 1-strata of $Y^{\mathrm{op}}(D)$ (the inner edges of the toric graph). The $\alpha_{i}$ are relative 2-homology classes corresponding to the outer edges with framing. The morphisms

$$
K_{Y \backslash D_{1} \cup \cdots \cup D_{l-1}} \stackrel{\pi}{\longrightarrow} Y \backslash D_{1} \cup \cdots \cup D_{l-1} \stackrel{i}{\longrightarrow} Y,
$$

with $\pi: K_{Y \backslash D_{1} \cup \cdots \cup D_{l-1}} \rightarrow Y \backslash D_{1} \cup \cdots \cup D_{l-1}$ the bundle projection and $i: Y \backslash D_{1} \cup \cdots \cup D_{l-1} \hookrightarrow Y$ the canonical open immersion, induce an injective homomorphism of 2-homology groups

$$
\iota_{o}: \mathrm{H}_{2}\left(Y \backslash D_{1} \cup \cdots \cup D_{l-1}, \mathbb{Z}\right) \hookrightarrow \mathrm{H}_{2}(Y, \mathbb{Z}) .
$$

In fact, by sending the generators to the corresponding subvarieties, we identify $\iota_{o}$ with a morphism

$$
\mathrm{H}_{2}\left(Y \backslash D_{1} \cup \cdots \cup D_{l-1}, \mathbb{Z}\right) \hookrightarrow \mathrm{A}_{1}(Y)
$$

\footnotetext{
${ }^{2}$ As discussed in more details in $\left.15, \S 1.4\right]$, point insertions and the log Calabi-Yau condition are both crucial assumptions allowing us to obtain the numerical log-local correspondence despite the general negative results of 8,55. In [15, §5], we gave a conceptual proof by degeneration of the numerical log-local correspondence for log Calabi-Yau surfaces with two boundary components. This completely general degeneration argument can be easily extended to the orbifold setting in any dimension and can in principle be used to determine when the numerical log-local correspondence holds and when correction terms are needed.
} 
The edge of the toric graph with framing $p_{i} / r_{i}$ corresponds to a toric $\left(\mathbb{A}^{1}\right)_{i}$ in $K_{Y \backslash D_{1} \cup \cdots \cup D_{l-1}}$ that meets the $i^{\text {th }}$ connected component $L_{i}$ of the toric Lagrangian $L$ in a non-trivial minimal $\left(S^{1}\right)_{i} \subset L_{i}$, i.e. $\left[\left(S^{1}\right)_{i}\right]$ generates $\mathrm{H}_{1}\left(L_{i}, \mathbb{Z}\right)$. Moreover, $\left[\left(S^{1}\right)_{i}\right]$ corresponds to the relative homology class

$$
\left[\left(\operatorname{disk} \subset\left(\mathbb{A}^{1}\right)_{i}, \partial \operatorname{disk}=\left(S^{1}\right)_{i}\right)\right] \in \mathrm{H}_{2}^{\mathrm{rel}}\left(Y^{\mathrm{op}}(D), L ; \mathbb{Z}\right) .
$$

The projection $\pi\left(\left(\mathbb{A}^{1}\right)_{i}\right)$ is compactified to an orbifold $\mathbb{P}_{r_{i}}^{1}$ in $Y$, with the added point a cyclic quotient singularity in $Y$ with isotropy group $\mathbb{Z} / r_{i} \mathbb{Z}$, see $\left[35\right.$, Section 3.11.3]. Furthermore, $r_{i}\left[\mathbb{P}_{r_{i}}^{1}\right]$ is a well-defined class in $\mathrm{H}_{2}(Y, \mathbb{Z})$. Following $\left[15\right.$, Definition 6.5], we send $\left[\left(S^{1}\right)_{i}\right]$ to $\left[\mathbb{P}_{r_{i}}^{1}\right]$. The latter is not in $\mathrm{H}_{2}(Y, \mathbb{Z})$, but it is in the Chow group $\mathrm{A}_{1}(Y)$. We will only be interested in winding numbers whose image lies in $\mathrm{H}_{2}(Y, \mathbb{Z})$. In particular, in the log-open correspondence of (2.18), only winding numbers that are multiples of the $r_{i}$ of their respective framings can be compared with curves classes on the log side, which introduces the correction term in (2.18) compared to [15. Conjecture 1.3]. In summary, we extend $\iota_{o}$ to an injective map

$$
\iota: \mathrm{H}_{2}\left(Y \backslash D_{1} \cup \cdots \cup D_{l-1}, \mathbb{Z}\right) \oplus \bigoplus_{i=1}^{l-1} \mathrm{H}_{1}\left(L_{i}, \mathbb{Z}\right) \hookrightarrow \mathrm{A}_{1}(Y)
$$

by positing that $\iota:\left[\left(S^{1}\right)_{i}\right] \mapsto\left[\mathbb{P}_{r_{i}}^{1}\right]$ for $i=1, \ldots, l-1$, and we will only be interested in curve classes $d \in \mathrm{H}_{2}(Y, \mathbb{Z})$ that admit an inverse $\iota^{-1}(d)$.

Theorem 2.2. For each of $Y(D)=\mathbb{P}(1, a, b), Y_{(a, b)}^{[2]}$ or $Y_{(a, b)}^{[3]}$, writing $f_{j}=\frac{p_{j}}{r_{j}}$ with $\left(p_{j}, r_{j}\right)=1$ for the framing of the $j^{\text {th }}$ Aganagic-Vafa orbi-brane in $Y^{\mathrm{op}}(D)$, we obtain

$$
\mathrm{O}_{\iota^{-1}(d)}\left(Y^{\mathrm{op}}(D)\right)(-\mathrm{i} \log q)=[1]_{q}^{l-2} \frac{(-1)^{d \cdot D_{l}-1}}{\left[d \cdot D_{l}\right]_{q}} \prod_{j=1}^{l-1} \frac{(-1)^{r_{j}\left(d \cdot D_{j}\right)-1}}{r_{j}\left(d \cdot D_{j}\right)} \mathrm{N}_{d}^{\log }(Y(D))(-\mathrm{i} \log q),
$$

as well as closed-form expressions of the invariants. The correction factor $r_{j}\left(d \cdot D_{j}\right)$ is the winding number around $L_{j}$.

In the genus zero limit $(q \rightarrow 1)$ Theorem 2.2 recovers a version of the numerical log/local correspondence of Theorem 2.1, with the genus zero open invariants equating the local invariants up to a factor:

$$
O_{0 ; \iota^{-1}(d)}\left(Y^{\mathrm{op}}(D)\right)=\prod_{j=1}^{l-1} \frac{(-1)^{d \cdot D_{j}\left(r_{j}-1\right)}}{r_{j}} N_{d}^{\mathrm{loc}}(Y(D))
$$

The additional normalisation factor as compared to the case of smooth varieties (where $r_{j}=1$ ), and especially the rescaling of the boundary circle classes by $r_{j}$ in the definition of $\iota$ are familiar in the relation of fractionally framed toric branes to enumerative invariants, and they match identically the correction factors relating open GW invariants of large $N$ Lagrangians of torus links from fractionally framed open GW invariants of toric orbi-branes; see [5, 18, 30, 35].

2.2.3. KP/LMOV/DT integrality. The next Theorem substantiates the expectation that 2.11)(2.12) are particular open BPS/LMOV partition functions, and in particular integral Laurent polynomials in $q^{1 / 2}$.

Theorem 2.3. Let $Y(D)$ be any of $\mathbb{P}(1, a, b), Y_{(a, b)}^{[2]}$ or $Y_{(a, b)}^{[3]}$. Then $\Omega_{d}(q) \in \mathbb{Z}\left[q^{ \pm 1 / 2}\right]$. 
In the genus zero limit, the combination of Theorems 2.1 and 2.2 gives

$$
\begin{aligned}
\Omega_{d}(Y(D)) & =\frac{1}{\prod_{i=1}^{l}\left(d \cdot D_{i}\right)} \sum_{k \mid d}(-1)^{d / k \cdot D+l} \frac{\mu(k)}{k^{4-2 l}} N_{d / k}^{\log }(Y(D)) \\
& =\sum_{k \mid d} \frac{\mu(k)}{k^{4-l}} \prod_{j=1}^{l-1} r_{j}(-1)^{d / k \cdot D_{j}\left(r_{j}+1\right)} O_{0 ; \iota^{-1}(d / k)}\left(Y^{\mathrm{op}}(D)\right) \\
& =\sum_{k \mid d} \frac{\mu(k)}{k^{4-l}} N_{d / k}^{\mathrm{loc}}(Y(D)) .
\end{aligned}
$$

It follows directly from Theorem 2.3 that $\Omega_{d}(Y(D)) \in \mathbb{Z}$. In particular, 2.21 implies that the unrefined BPS invariants $\Omega_{d}(Y(D))$ coincide with an orbifold generalisation of the conjecturally integral invariants $\mathrm{KP}_{d}\left(E_{Y(D)}\right)$ of the local orbifold surfaces $\mathbb{P}(1, a, b), Y_{(a, b)}^{[2]}$ and $Y_{(a, b)}^{[3]}$ introduced by Klemm-Pandharipande for $l=2$ in [46] and by Ionel-Parker in [45] for smooth varieties. Theorem 2.3 implies then immediately their integrality in the generalised orbifold context of this paper.

Finally, when $l=2$ and the Lagrangians of $Y^{\mathrm{op}}(D)$ are integrally framed, the integrality statement above is also a consequence of the following statement, which is a direct consequence of the stripsquiver correspondence of [57] (see in particular [15, Theorem 7.3])

Theorem 2.4. Let $Y(D)=\mathbb{P}(1, a, b)$ or $Y(D)=Y_{(a, b)}^{[2]}$ with either $a=1$ or $b=1$. Then there exists a symmetric quiver $\mathrm{Q}(Y(D))$ with $\chi(Y)-1$ vertices and a lattice isomorphism $\kappa: \mathbb{Z}(\mathrm{Q}(Y(D)))_{0} \stackrel{\sim}{\rightarrow}$ $\mathrm{H}_{2}(Y, \mathbb{Z})$ such that

$$
\mathrm{DT}_{d}^{\mathrm{num}}(\mathrm{Q}(Y(D)))=\left|\Omega_{\kappa(d)}(Y(D))+\sum_{i} \alpha_{i} \delta_{d, v_{i}}\right|
$$

with $\alpha_{i} \in\{0,1\}$. In particular, $\Omega_{d}(Y(D)) \in \mathbb{Z}$.

2.2.4. Geometric motivation. In the smooth case, the rational underpinning of the web of correspondences of the previous Section was described in [15, Section 1.4]; we recall it briefly here. Let $Y\left(D=D_{1}+\cdots+D_{l}\right)$ be a log smooth log Calabi-Yau surface and let $d$ be a curve class such that $d \cdot D_{l}>0$. If $D_{l}$ is nef, or more generally if $d$ is $D_{l}$-convex 15 , Section 1.4.1], then by the main result of [36] the genus $0 \log$ Gromov-Witten $(\mathrm{GW})$ theory of maximal tangency of $\left(Y, D_{l}\right)$ is equivalent to the genus 0 local Gromov-Witten theory of $\operatorname{Tot}\left(\mathcal{O}\left(-D_{l}\right) \rightarrow S\right)$. This correspondence extends to adding maximal tangency conditions along the divisors $D_{j}$ on $S$, resp. $\left.\mathcal{O}\left(-D_{l}\right)\right|_{D_{j}}$ on $\operatorname{Tot}\left(\mathcal{O}\left(-D_{l}\right) \rightarrow S\right)$ : in other words, there is a duality between imposing a maximal tangency condition along $D_{l}$ and twisting by $\mathcal{O}\left(-D_{l}\right)$. This insight forms the basis of many subsequent works 8 10, $14,15,23,25,34,55,61,62$.

An important question is the extent to which the above generalises to simple normal crossings divisors, particularly in light of a counter-example for the log-local correspondence which was given in the log Fano case in [55]. On the other hand there is growing evidence that the equivalence could persist in the log Calabi-Yau case in the stationary sector, see [14, Theorem 3.4], [15, Theorem 5.1], and the geometric argument of [15, Section 5] which is further amenable to treating the log-local 
correspondence for log smooth log Calabi-Yau varieties in any dimension. Theorem 2.1 further corroborates this expectation in the orbifold context.

The log-open correspondence of Section 2.2.2 stems from one of the realisations of [15], whereby a maximal tangency condition along $D_{j}$ is proposed to be heuristically replaceable by an open condition along a Lagrangian $L_{j}$ near $D_{j}$. For log CY surfaces $\left(Y, D_{1}+\cdots+D_{l}\right)$ this entails a precise correspondence at the level of logarithmic and open invariants, as we explain in [15, Section 1.4.2]: one first employs the log-local correspondence above for the a single irreducible component $D_{l}$ to twist $Y$ by $\mathcal{O}\left(-D_{l}\right)$, and then consider Lagrangians $L_{j}$ near $\left.\mathcal{O}\left(-D_{l}\right)\right|_{D_{j}}, j=1, \ldots, l-1$. It is shown in 15 that under suitable conditions these Lagrangians are singular Harvey-Lawson (AganagicVafa) branes, for which a rigorous construction of the open invariants exists [50]. The arguments of 15. Section 1.4.2 and Construction 6.4], adapted to the orbifold context, lead then to the log-open relations 2.18) and (2.19) above.

Finally, parallel to similar expectations for other enumerative theories, underlying the all genus Gromov-Witten invariants of $Y(D)$ are the BPS invariants defined by (2.11). These are conjectured to be integer-valued Laurent polynomials in $q^{1 / 2}$, which we prove for Examples I-III in Theorem 2.3 . When $l=2$, it was proposed in [15, Section 1.4.3] that the unrefined $(q \rightarrow 1)$ limit of these BPS invariants should recover, up to signs, the Donaldson-Thomas invariants of a symmetric quiver. This is suggested by the conjectured relation of Gopakumar-Vafa invariants with sheafcounting theories on 4-folds 19,20 and their connection, for local surfaces, to moduli of quiver representations highlighted in [15. Section 1.4.3], and simultaneously by a joint use of the 'branesquivers' correspondence of [33, 47] and the log-open correspondence above. It was proposed in 15. Section 1.5.4] that the same chain of ideas may apply in the orbifold context too: Theorem 2.4 indeed establishes this expectation for the $l=2$ examples of this paper.

\subsection{The techniques.}

2.3.1. Scattering diagrams. Our main tool for the computations of $\mathrm{N}_{d}^{\log }(Y(D))$ are multiplications of quantum broken lines in the quantum scattering diagrams of $12,13,29,51$. In the classical limit, this is treated in $39,40,42$ in dimension 2 and in full generality in 41,43 . The quantum scattering diagram associated to $Y(D)$ consists of an affine integral manifold $B$ and a collection of walls $\mathfrak{d}$ with wall-crossing functions $f_{\mathfrak{b}}$. Here we content ourselves to give a brief overview, referring to 15. Section 4.2] for details of the construction, the wall-crossing algorithm, broken lines and their

multiplication. In particular, 15. Proposition 4.2] explains how to extract the $\mathrm{N}_{d}^{\log }(Y(D))$ from structural coefficients of the multiplication of theta functions.

As a topological manifold, $B$ is homeomorphic to $\mathbb{R}^{2}$. It comes with some distinguished integral rays $\rho_{1}, \ldots, \rho_{l+r}$ emanating from the origin. Up to reordering, $\rho_{1}, \ldots, \rho_{l}$ will correspond to $D_{1}, \ldots, D_{l}$. The collection of all rays forms a fan with associated toric variety $\bar{Y}\left(\bar{D}=D_{\rho_{1}}+\cdots+D_{\rho_{l+r}}\right)$ for the toric prime divisors $D_{\rho_{1}}, \ldots, D_{\rho_{l+r}}$ of $\bar{Y}$. Some of these rays, say $\rho_{j_{1}}, \ldots, \rho_{j_{s}}$ with possible repetitions, have focus-focus singularities on them. For our purposes, we perturb these away from their rays, which simply means the creation of lines parallel to the rays carrying the focus-focus singularities. These walls are decorated with wall-crossing functions. When two walls meet, there 
is scattering resulting in the creation of new walls carrying wall-crossing functions themselves. For our examples, there will only be "simple" scattering.

For each focus-focus singularity on a line parallel to $\rho_{j_{i}}$, we blow up a smooth point of $D_{\rho_{j_{i}}}$. Taken together, this yields a toric model

$$
\pi:(\widetilde{Y}, \widetilde{D}) \longrightarrow(\bar{Y}, \bar{D}) .
$$

This means that there is a birational map

$$
\varphi:(\tilde{Y}, \widetilde{D}) \longrightarrow(Y, D)
$$

that is a sequence of blow ups at codimension 2 strata of the boundary. By $[3],(\widetilde{Y}, \widetilde{D})$ and $(Y, D)$ have the same log GW theory.

Then, $B$ has integral asymptotic directions that correspond to weighted blow ups of $(\bar{Y}, \bar{D})$. Theta functions (which are sections of an ample line bundle on the mirror family) correspond to asymptotic directions. Their values on open subsets of the mirror corresponding to the chambers of the scattering diagram are given by the sums of the end-coefficients of broken lines coming from the corresponding asymptotic directions. The broken lines can bend when crossing walls picking up contributions from the wall-crossing functions.

Multiplying broken lines together corresponds to creating tropical curves with the correct weights (=intersection multiplicities) with a selection of (possibly weighted blow ups of) boundary divisors. The balancing condition has to be satisfied at each vertex except at the focus-focus singularities, which are seen as sources of Maslov index 0 disks. These tropical curves furthermore are weighted by contributions coming from the wall-crossing. Summing the weights of the tropical curves then calculates the $\mathrm{N}_{d}^{\log }(Y(D))$ as described in [15, Proposition 4.2].

2.3.2. Local mirror symmetry. Since $Y$ is a projective toric surface for all of Examples I-III, we can avail ourselves of Givental-type mirror theorems to determine $(2.4)-(2.5)$. Let $T \simeq\left(\mathbb{C}^{\star}\right)^{l}$ be the torus action on $E_{Y(D)}$ covering the trivial action on the zero section $\iota: Y \hookrightarrow E_{Y(D)}$. Fix $\left\{\varphi_{\alpha}\right\}_{\alpha=0}^{\chi(Y)-1}$ a $\mathrm{H}(B T)$-basis of $\mathrm{H}_{T}\left(E_{Y(D)}\right)$ given by lifts to $T$-equivariant cohomology of classes $\phi_{\alpha} \in \mathrm{H}(Y)$ with $\operatorname{deg} \phi_{\alpha} \leq \operatorname{deg} \phi_{\alpha+1}$, and for $\theta, \chi \in \mathrm{H}_{T}\left(E_{Y(D)}\right)$ denote $\eta_{Y(D)}(\theta, \chi)$ the T-equivariant Poincaré pairing on $E_{Y(D)}$,

$$
\eta_{Y(D)}(\theta, \chi):=\int_{Y} \frac{\iota^{*} \theta \cup \iota^{*} \chi}{\cup_{m=1}^{l} \mathrm{e}_{T}\left(\mathcal{O}_{Y}\left(-D_{m}\right)\right)} .
$$

In terms of the small $T$-equivariant $J$-function of $E_{Y(D)}$,

$$
J_{\text {small }}^{Y(D)}(t, z):=z \mathrm{e}^{\sum_{i=1}^{\rho(Y)} t_{i} \varphi_{i} / z}\left(1+\sum_{d \in \operatorname{NE}(Y)} \sum_{\alpha, \beta} \eta_{Y(D)}^{-1}\left(\varphi_{\alpha}, \varphi_{\beta}\right) \mathrm{e}^{t \cdot d}\left\langle\frac{\varphi_{\alpha}}{z\left(z-\psi_{1}\right)}\right\rangle_{0,1, d}^{E_{Y(D)}} \varphi_{\beta}\right),
$$

(2.5) is given by

$$
N_{d}^{\mathrm{loc}, \psi}(Y(D))=\left[z^{l-1} \mathrm{e}^{t \cdot d}\right] \eta_{Y(D)}\left(\mathrm{pt}, J_{\mathrm{small}}^{Y(D)}(t, z)\right)
$$

where for a ring $R$ and $f\left(x_{1}, \ldots, x_{n}\right)=\sum_{i_{1}, \ldots, i_{n} \geq 0} c_{i_{1} \ldots i_{n}} x_{1}^{i_{1}} \ldots x_{n}^{i_{n}} \in R\left[\left[x_{1}, \ldots, x_{n}\right]\right]$ a formal power series with $R$-coefficients, we write $\left[\prod_{l=1}^{n} x_{l}^{i_{l}}\right] f(x)$ for the formal Taylor coefficient $c_{i_{1}, \ldots, i_{n}}$ 
in $\left(x_{1}, \ldots, x_{n}\right)$, and we employed the usual correlator notation for GW invariants,

$$
\left\langle\tau_{1} \psi_{1}^{k_{1}}, \ldots, \tau_{n} \psi_{n}^{k_{n}}\right\rangle_{0, n, d}^{E_{Y(D)}}:=\int_{\left[\overline{\mathrm{M}}_{0, m}\left(E_{Y(D)}, d\right)\right]^{\mathrm{vir}}} \prod_{i} \operatorname{ev}_{i}^{*}\left(\tau_{i}\right) \psi_{i}^{k_{i}}
$$

We compute the r.h.s. of $(2.27)$ using the Coates-Givental-Tseng twist $26,28,37,38,60$ at the $J$-function level, equating $J_{\text {small }}^{Y(D)}(t, z)$, up to a mirror map $t \mapsto t(y)$, to an explicit generalised hypergeometric series $I^{Y(D)}(y, z)$, which is in turn read off from the fan of $E_{Y(D)} 22,26,27,38$. In all of Examples I-III we will have $t=\log y$, and thus

$$
N_{d}^{\mathrm{loc}, \psi}(Y(D))=\left[z^{l-1} y^{d}\right] \eta_{Y(D)}\left(\mathrm{pt}, I^{Y(D)}(y, z)\right) .
$$

To compute (2.4), we use part of a reconstruction theorem due to Boris Dubrovin [31, Lecture 6], combined with the vanishing of quantum corrections to certain products in quantum cohomology. Recall that the components of the big $J$-function,

$$
J_{\text {big }}^{Y(D)}(\tau, z):=z+\tau+\sum_{\mathrm{d} \in \mathrm{NE}(Y)} \sum_{n \in \mathbb{Z}^{+}} \frac{1}{n !} \sum_{\alpha, \beta} \eta_{Y(D)}^{-1}\left(\varphi_{\alpha}, \varphi_{\beta}\right)\left\langle\tau, \ldots, \tau, \frac{\varphi_{\alpha}}{z-\psi_{1}}\right\rangle_{0, n+1, d}^{E_{Y(D)}} \varphi_{\beta},
$$

form a basis of flat co-ordinates for the Dubrovin connection,

$$
z \nabla_{\theta} \nabla_{\chi} J_{\text {big }}^{Y(D)}(\tau, z)=\nabla_{\theta \star_{\tau} \chi} J_{\text {big }}^{Y(D)}(\tau, z),
$$

with $\tau \in \mathrm{H}_{T}\left(E_{Y(D)}\right)$ and $\star_{\tau}$ the big quantum cohomology product, the restriction to small quantum cohomology being $\tau \rightarrow \sum_{i=1}^{\rho(Y)} t_{i} \varphi_{i}$. Suppose now that there exist numbers $\varpi_{i j}^{Y(D)}, i, j=1, \ldots, \rho(Y)$ such that

$$
\left.\sum_{i, j=1}^{\rho(Y)} \varpi_{i j}^{Y(D)} \varphi_{i} \star_{\tau} \varphi_{j}\right|_{\tau \rightarrow \sum_{i=1}^{\rho(Y)} t_{i} \varphi_{i}}=\sum_{i, j=1}^{\rho(Y)} \varpi_{i j}^{Y(D)} \varphi_{i} \cup \varphi_{j}=\mathrm{pt}
$$

Then,

$$
z \sum_{i, j=1}^{\rho(Y)} \varpi_{i j}^{Y(D)} \partial_{t_{i} t_{j}}^{2} J_{\mathrm{small}}^{Y(D)}(t, z)=\left.\nabla_{\mathrm{pt}} J_{\mathrm{big}}^{Y(D)}(\tau, z)\right|_{\tau \rightarrow \sum_{i=1}^{\rho(Y)} t_{i} \varphi_{i}}
$$

from which we deduce

$$
\begin{aligned}
N_{d}^{\mathrm{loc}}(Y(D)) & =\left[z^{l-1} y^{d}\right] \eta_{Y(D)}\left(\mathrm{pt}, \sum_{i, j=1}^{\rho(Y)} \varpi_{i j}^{Y(D)} q_{i} q_{j} \partial_{q_{i} q_{j}}^{2} I^{Y(D)}(y, z)\right) \\
& =\left(\sum_{i, j=1}^{\rho(Y)} \varpi_{i j}^{Y(D)} d_{i} d_{j}\right) N_{d}^{\mathrm{loc}, \psi}(Y(D))
\end{aligned}
$$

2.3.3. The topological vertex. The connected generating functions $\mathcal{O}_{\vec{\mu}}\left(Y^{\mathrm{op}}(D)\right)(Q, \hbar)$ of open $\mathrm{GW}$ invariants in the 'winding-number basis' of [50],

$$
\mathcal{O}_{\vec{\mu}}\left(Y^{\mathrm{op}}(D)\right)(Q, \hbar):=\sum_{\beta} \mathrm{O}_{\beta, \vec{\mu}}\left(Y^{\mathrm{op}}(D)\right)(\hbar) Q^{\beta}
$$




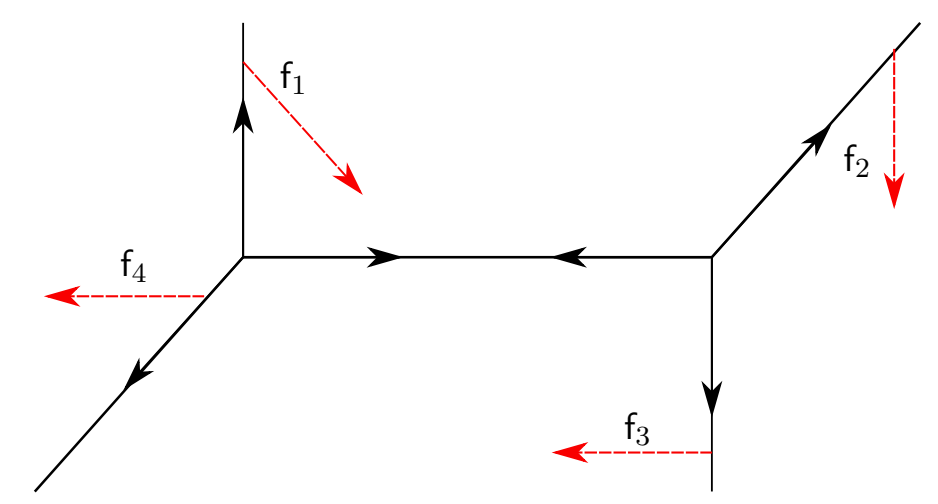

FiguRE 2.1. The toric graph of the resolved conifold with four outer framed Lagrangians.

can be reconstructed from the disconnected generating functions $\mathcal{Z}_{\vec{\mu}}\left(Y^{\mathrm{op}}(D)\right)$ and $\mathcal{W}_{\vec{\mu}}\left(Y^{\mathrm{op}}(D)\right)$ in the 'winding number' and 'representation' bases defined by

$$
\begin{aligned}
\exp \left[\sum_{\vec{\mu} \in(\mathcal{P})^{l-1}} \mathcal{O}_{\vec{\mu}}\left(Y^{\mathrm{op}}(D)\right)(Q, \hbar) \mathrm{x}_{\vec{\mu}}\right] & =: \sum_{\vec{\mu} \in(\mathcal{P})^{l-1}} \mathcal{Z}_{\vec{\mu}}\left(Y^{\mathrm{op}}(D)\right)(Q, \hbar) \overrightarrow{\mathrm{x}}_{\vec{\mu}}, \\
& =: \sum_{\vec{\mu}, \vec{\nu} \in(\mathcal{P})^{l-1}} \prod_{i=1}^{l-1} \frac{\chi_{\nu_{i}}\left(\mu_{i}\right)}{z_{\mu_{i}}} \mathcal{W}_{\vec{\nu}}\left(Y^{\mathrm{op}}(D)\right)(Q, \hbar) \mathrm{x}_{\vec{\mu}}
\end{aligned}
$$

In the equation above, $\mathcal{P}$ denotes the set of partitions and $\chi_{\alpha}(\beta)$ denotes the value of the irreducible character of the symmetric group $S_{|\alpha|}$ on the conjugacy class labelled by the partition $\beta$.

The theory of the topological vertex consists of a glueing procedure to algorithmically compute $\mathcal{W}_{\vec{\nu}}\left(Y^{\mathrm{op}}(D)\right)$ from the representation-basis generating functions of toric Lagrangian triples $Y^{\mathrm{op}}(D)_{i}$, $i=1,2$ openly embedded into $Y(D)$. We content ourselves to state the consequences of the glueing algorithm in the two cases needed for for the study of Examples I-III, referring the reader to 15. Sec. 6.1.2] for a complete account. If $Y^{\mathrm{op}}(D)=\left(\operatorname{Tot}\left(\mathcal{O}_{\mathbb{P}^{1}}^{\oplus 2}(-1)\right), L, \mathrm{f}\right)$ is the resolved conifold with $L$ the disconnected union of four outer branes in representations $\mu_{1}, \ldots, \mu_{4}$ and framing shifts $\mathrm{f}=\left(f_{1}, \ldots, f_{4}\right)$ as in Figure 2.1, we have

$$
\begin{aligned}
& \mathcal{W}_{\mu_{1}, \mu_{2}, \mu_{3}, \mu_{4}}\left(\operatorname{Tot}\left(\mathcal{O}_{\mathbb{P}^{1}}^{\oplus 2}(-1)\right), L, f\right)(Q, \hbar)= \\
= & \sum_{\nu \in \mathcal{P}} \mathcal{W}_{\mu_{1}, \mu_{2}, \nu}\left(\mathbb{C}^{3},\left(L_{1}, L_{2}, L_{0}\right),\left(f_{1}, f_{2}, f_{0}\right)\right)(\hbar)(-Q)^{|\nu|} \mathcal{W}_{\nu^{T}, \mu_{3}, \mu_{4}}\left(\mathbb{C}^{3}, L,\left(-f_{0}, f_{3}, f_{4}\right)\right)(\hbar),
\end{aligned}
$$

and if $Y^{\mathrm{op}}(D)=\left(\mathbb{C}^{3}, L, \mathrm{f}\right)$ is the affine space with three outer branes in representations $\mu_{1}, \ldots, \mu_{3}$ and framing shifts $\mathrm{f}=\left(f_{1}, \ldots, f_{3}\right)$ as in Figure 2.2 (the framed 3-legged vertex), we have [4,53]

$$
\mathcal{W}_{\mu_{1}, \mu_{2}, \mu_{3}}\left(\mathbb{C}^{3}, L, \mathrm{f}\right)(\hbar)=q^{\kappa\left(\mu_{1}\right) / 2} \prod_{i=1}^{3} q^{f_{i} \kappa\left(\mu_{i}\right) / 2}(-1)^{f_{i}\left|\mu_{i}\right|} \sum_{\delta \in \mathcal{P}} s_{\frac{\mu_{1}^{t}}{\delta}}\left(q^{\rho+\mu_{3}}\right) s_{\frac{\mu_{2}}{\delta}}\left(q^{\rho+\mu_{3}^{t}}\right) s_{\mu_{3}}\left(q^{\rho}\right)
$$




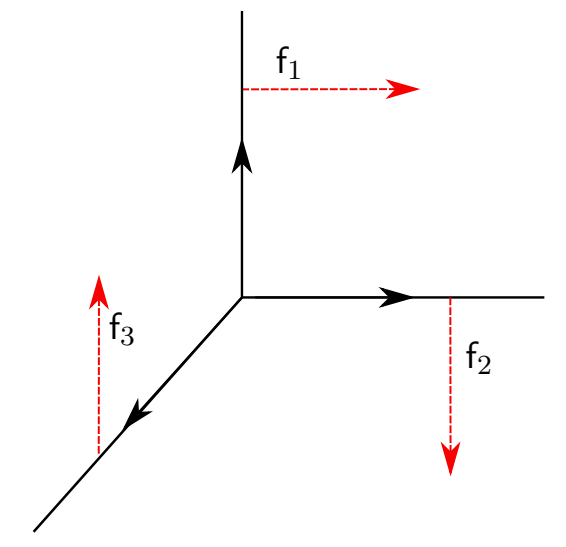

FiguRE 2.2. The toric graph of the framed 3-legged vertex.

where $s_{\mu}\left(q^{\rho+\alpha}\right)$ denotes the principally-specialised $\alpha$-shifted Schur function in the representation of GL $(\infty)$ labelled by $\mu$ (see [15, Appendix C] for details), $\kappa(\mu)$ is its second Casimir invariant of the partition $\mu$ normalised as $\kappa((1))=0$, and again $q=\mathrm{e}^{\mathrm{i} \hbar}$.

\section{Example I}

Let $a, b$ be coprime positive integers. Then $\mathbb{P}(1, a, b)$ has toric divisors $D_{(-1,0)}, D_{(0,-1)}$ and $D_{(b, a)}$ with relations

$$
D_{(-1,0)} \sim b D_{(b, a)}, \quad D_{(0,-1)} \sim a D_{(b, a)}
$$

intersections

$$
D_{(-1,0)} \cdot D_{(0,-1)}=1, \quad D_{(0,-1)} \cdot D_{(b, a)}=\frac{1}{b}, \quad D_{(-1,0)} \cdot D_{(b, a)}=\frac{1}{a},
$$

and self-intersections

$$
D_{(-1,0)}^{2}=\frac{b}{a}, \quad D_{(0,-1)}^{2}=\frac{a}{b}, \quad D_{(b, a)}^{2}=\frac{1}{a b} .
$$

To obtain a $\log$ smooth nef $\log$ Calabi-Yau surface, we choose $D_{1}=D_{(b, a)}$ and $D_{2}$ a smooth element of the linear system of $D_{(-1,0)}+D_{(0,-1)}$ so that

$$
D_{1}^{2}=\frac{1}{a b}, \quad D_{2}^{2}=\frac{(a+b)^{2}}{a b}
$$

Writing $D=D_{1}+D_{2}$, the topological Euler characteristic of the complement of $D$ is

$$
\chi(\mathbb{P}(1, a, b) \backslash D)=1 .
$$

Denote by $H$ the effective generator of $\mathrm{H}_{2}(\mathbb{P}(1, a, b), \mathbb{Z})$. Notice that $H^{2}=a b, D_{1} \sim \frac{1}{a b} H$ and $D_{2} \sim \frac{a+b}{a b} H$. Consequently, for a curve class $d=d H$,

$$
d \cdot D_{1}=d, \quad d \cdot D_{2}=d(a+b) .
$$


3.1. Local GW invariants. Let $T \simeq\left(\mathbb{C}^{\star}\right)^{2} \circlearrowright E_{\mathbb{P}(1, a, b)}$ be the fibre-wise action on $E_{\mathbb{P}(1, a, b)}$, and denote by $\lambda_{i}, i=1,2$ its equivariant parameters. The inverse of the Gram matrix of the $T$ equivariant Poincaré pairing in the basis $\left\{1, H, H^{2}\right\}$ reads

$$
\eta_{\mathbb{P}(1, a, b)}^{-1}=\left(\begin{array}{ccc}
0 & 0 & \frac{\lambda_{1} \lambda_{2}}{a b} \\
0 & \frac{\lambda_{1} \lambda_{2}}{a b} & -\frac{a \lambda_{1}+b \lambda_{1}+\lambda_{2}}{a^{2} b^{2}} \\
\frac{\lambda_{1} \lambda_{2}}{a b} & -\frac{a \lambda_{1}+b \lambda_{1}+\lambda_{2}}{a^{2} b^{2}} & \frac{a+b}{a^{3} b^{3}}
\end{array}\right)
$$

From [38, Thm 0.1] and 26, Thm 4.6], the $T$-equivariant $I$-function of $E_{\mathbb{P}(1, a, b)}$ is

$$
I^{\mathbb{P}(1, a, b)}(y, z)=z y^{H / z} \sum_{d \geq 0} y^{d} \frac{\prod_{m=0}^{d-1}\left(\lambda_{1}-H /(a b)-m z\right) \prod_{m=0}^{(a+b) d-1}\left(\lambda_{2}-(a+b) H /(a b)-m z\right)}{\prod_{m=1}^{d}(H /(a b)+m z) \prod_{m=1}^{a d}(H / b+m z) \prod_{m=1}^{b d}(H / a+m z)} .
$$

We have

$$
I^{\mathbb{P}(1, a, b)}(y, z)=z+(\log y) H+\mathcal{O}\left(\frac{1}{z}\right),
$$

and therefore, as alluded to in Section 2.3.2, the mirror map is trivial,

$$
J_{\text {small }}^{\mathbb{P}(1, a, b)}(t, z)=I^{\mathbb{P}(1, a, b)}\left(e^{t}, z\right) .
$$

Since $l=2$ and thus $N_{d}^{\text {loc, } \psi}(\mathbb{P}(1, a, b))=N_{d}^{\text {loc }}(\mathbb{P}(1, a, b))$, 2.27) gives

$$
N_{d}^{\mathrm{loc}}(\mathbb{P}(1, a, b))=\frac{1}{a b} \sum_{i \in\{0,1,2\}}\left(\eta_{\mathbb{P}(1, a, b)}\right)_{2, i}\left[H^{i} z^{-1} y^{d}\right] I^{\mathbb{P}(1, a, b)}(y, z),
$$

where, from (3.8), we have

$$
\left[z^{-1} y^{d}\right] I^{\mathbb{P}(1, a, b)}(y, z)=\frac{(-1)^{d(a+b+1)} \Gamma((a+b) d)\left(H-a b \lambda_{1}\right)\left(H(a+b)-a b \lambda_{2}\right)}{a^{2} b^{2} d \Gamma(a d+1) \Gamma(b d+1)},
$$

and we have used that $H^{2}=(a b)$ pt. Combining (3.7), 3.11) and (3.12) finally yields

$$
N_{d}^{\mathrm{loc}}(\mathbb{P}(1, a, b))=\frac{(-1)^{d(a+b+1)}}{d^{2}(a+b)}\left(\begin{array}{c}
(a+b) d \\
a d
\end{array}\right) .
$$

\subsection{Log GW invariants.}

Proposition 3.1. Let $a, b$ be coprime positive integers. Then

$$
\mathrm{N}_{d}^{\log }(\mathbb{P}(1, a, b))(\hbar)=\left[\begin{array}{c}
(a+b) d \\
a d
\end{array}\right]_{q} .
$$

In the $\hbar \rightarrow 0(q \rightarrow 1)$ limit, this recovers the log-local correspondence of Theorem 2.1 for $Y(D)=$ $\mathbb{P}(1, a, b)$.

Proof. We follow the computational technique sketched out in Section 2.3.1, and given in full detail in 15 , Section 4.2]. We start by finding a toric model for $\left(\mathbb{P}(1, a, b), D_{1}+D_{2}\right)$. We view $\mathbb{P}(1, a, b)$ as given by the fan generated by $(-1,0),(0,-1)$ and $(b, a)$. We add a ray in the direction $(-1,1)$ which yields a new divisor $D_{3}$ and a birational map

$$
\varphi:\left(\widetilde{\mathbb{P}(1, a, b)},\left(D_{1}-D_{3}\right)+\left(D_{2}-D_{3}\right)+D_{3}\right) \rightarrow\left(\mathbb{P}(1, a, b), D_{1}+D_{2}\right) .
$$




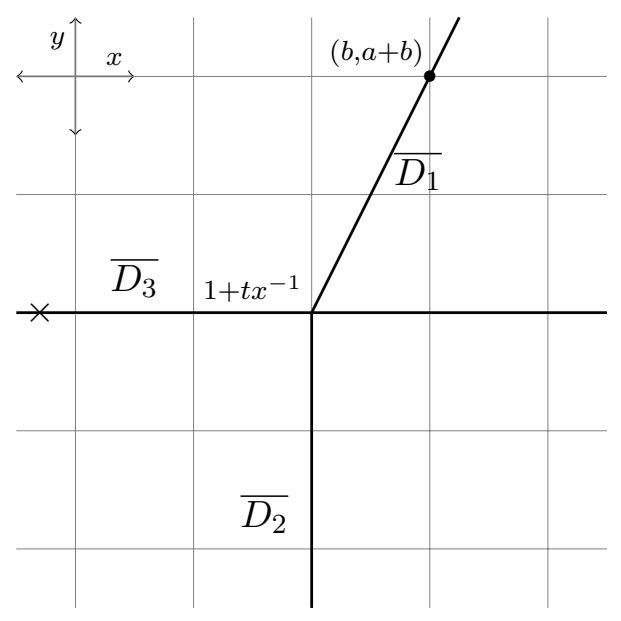

Figure 3.1. The toric model of $\mathbb{P}(1, a, b)$.

Then the proper transform of $D_{(-1,0)}$ is a $(-1)$-curve, which we contract:

$$
\pi:\left(\widetilde{\mathbb{P}(1, a, b)},\left(D_{1}-D_{3}\right)+\left(D_{2}-D_{3}\right)+D_{3}\right) \rightarrow\left(\overline{\mathbb{P}(1, a, b)}, \overline{D_{1}}+\overline{D_{2}}+\overline{D_{3}}\right) .
$$

The complement of the proper transform of $\bar{D}=\overline{D_{1}}+\overline{D_{2}}+\overline{D_{3}}$ now has Euler characteristic 0, hence is $\left(\mathbb{C}^{*}\right)^{2}$, therefore the variety is toric. We apply the $\mathrm{SL}_{2}(\mathbb{Z})$ transformation given by $\left(\begin{array}{ll}1 & 0 \\ 1 & 1\end{array}\right)$ to the fan and obtain the toric model of Figure 3.1. The toric surface $\overline{\mathbb{P}(1, a, b)}$ is the weighted projective space $\mathbb{P}(1, a+b, b)$. $\pi$ is determined by blowing up a smooth point on the divisor $\overline{D_{3}}$. This yields a focus-focus singularity on the ray directed by $(-1,0)$ corresponding to $\overline{D_{3}}$.

Next we multiply the theta functions corresponding to $D_{1}$ and $D_{2}$ and extract the identity component. To do so, we consider all the ways of combining two broken lines, one coming from the direction $D_{1}$ with weight $d \cdot D_{1}$, one coming from the direction $D_{2}$ with weight $d \cdot D_{2}$, at a fixed point $p$, so that they are opposite at $p$. Then the desired log Gromov-Witten invariant is the sum of the product over the end-coefficients of all possible ways of combining two broken lines in that way. There is only one configuration that solves this problem. This starts with a broken line $\beta_{1}$ coming from the $D_{1}$-direction carrying the monomial $\left(x^{b} y^{a+b}\right)^{d \cdot D_{1}}=x^{b d} y^{(a+b) d}$ and a broken line $\beta_{2}$ coming from the $D_{2}$-direction carrying the monomial $\left(y^{-1}\right)^{d \cdot D_{2}}=y^{-(a+b) d}$. The focus-focus singularity produces a wall with wall-crossing function $1+t x^{-1}$, see [15, Section 4.2]. When $\beta_{2}$ crosses this wall, it picks up a contribution from the wall-crossing function, see [15, Section 4.2]. In order to be opposite to $\beta_{1}$ at $p, \beta_{2}$ picks up the wall-crossing contribution that has degree $-b d$ in $x$. In fact, the resulting product of broken lines needs to respect the intersection profile of $d$. Since the focus-focus singularity corresponds to $D_{(-1,0)}$ and $d \cdot D_{(-1,0)}=b d$, the product of the end-coefficients of $\beta_{1}$ and $\beta_{2}$ needs to have degree $b d$ in $t$, see [15, Section 4.2]. Either condition determines the wall-crossing as sketched in Figure 3.2 and $\beta_{2}$ picks up the $q$-binomial coefficient $\left[\begin{array}{c}(a+b) d \\ b d\end{array}\right]_{q}=\left[\begin{array}{c}(a+b) d \\ a d\end{array}\right]_{q}$ which is $\mathrm{N}_{d}^{\log }(\mathbb{P}(1, a, b))(q)$ by 15 , Proposition 4.2]. 


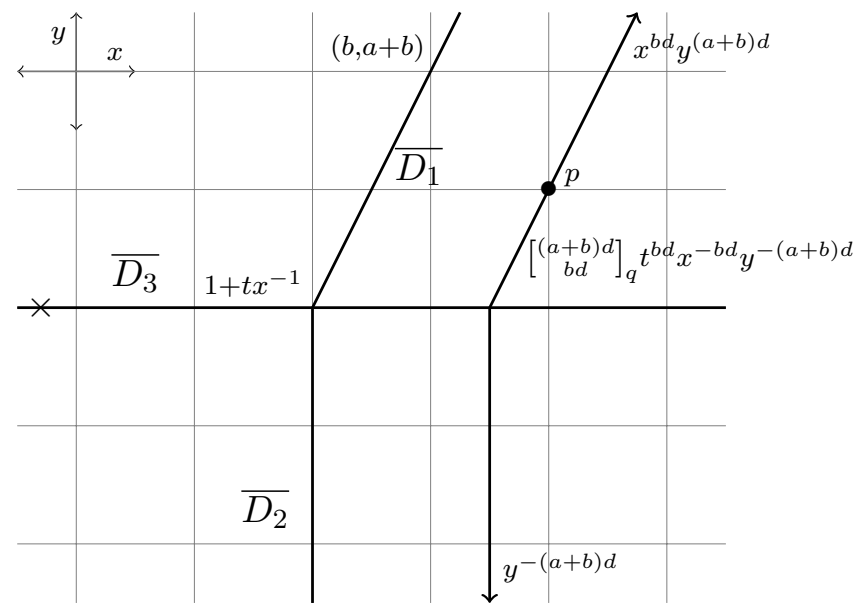

FiguRE 3.2. Scatt $\mathbb{P}(1, a, b)$

3.3. Open GW invariants. By [15, Construction 6.4], $(\mathbb{P}(1, a, b))^{\text {op }}$ is a toric Lagrangian triple $(X, L, \mathrm{f})$ depicted by the toric graph in Figure 3.3 . In particular, deleting $D_{1}=D_{(b, a)}$ in $\mathbb{P}(1, a, b)$ gives two dimensional affine space $\mathbb{C}^{2}$, and therefore $X=K_{\mathbb{P}(1, a, b) \backslash D_{(b, a)}}=K_{\mathbb{C}^{2}} \simeq \mathbb{C}^{3}$. The compactification to $K_{\mathbb{P}(1, a, b)}$ induces a framing on an outer Lagrangian $L$ on $\mathbb{C}^{3}$ shifted by $b / a$ with respect to the canonical framing, and the finite index morphism $\iota: \mathrm{H}_{2}^{\text {rel }}\left(\mathbb{C}^{3}, L, \mathbb{Z}\right) \simeq \mathrm{H}_{1}(L, \mathbb{Z}) \rightarrow$ $A_{1}(\mathbb{P}(1, a, b))$ is defined by $\left[S^{1}\right] \rightarrow\left[D_{(-1,0)}\right]=H / a$. In particular, the winding number $a d$ around $L$ corresponds to the curve class $d H$.

The all-genus, 1-hole open GW generating function of $(\mathbb{P}(1, a, b))^{\text {op }}$ at winding $j=a d$ is, by (2.35) and $(2.38)$ :

$$
\begin{aligned}
\mathrm{O}_{j}\left((\mathbb{P}(1, a, b))^{\mathrm{op}}\right) & =\sum_{s=0}^{j-1} \frac{(-1)^{s}}{j} \mathcal{W}_{\left(j-s, 1^{s}\right), \emptyset, \emptyset}\left(\mathbb{C}^{3}, L, \mathrm{f}\right) \\
& =\sum_{s=0}^{j-1} \frac{(-1)^{s}}{j} q^{b / a \kappa\left(\left(j-s, 1^{s}\right) / 2\right)}(-1)^{b / a j} s_{\left(j-s, 1^{s}\right)}\left(q^{\rho}\right),
\end{aligned}
$$

where $\left(j-s, 1^{s}\right)$ denotes a partition represented by a hook Young diagram with $j$ boxes and $s+1$ rows. Using the hook formula for Schur functions [59],

we get

$$
s_{\left(j-s, 1^{s}\right)}\left(q^{\rho}\right)=\frac{q^{\frac{1}{2}\left(\begin{array}{c}
j \\
2
\end{array}\right)-\frac{j s}{2}}}{[j]_{q}[j-s]_{q} ![s]_{q} !},
$$

$$
\begin{aligned}
\mathrm{O}_{j}\left((\mathbb{P}(1, a, b))^{\mathrm{op}}\right) & =\frac{(-1)^{b / a j} q^{\left(\frac{b}{a}+\frac{1}{2}\right)\left(\begin{array}{l}
j \\
2
\end{array}\right)}}{j[j]_{q}} \sum_{s=0}^{d-1} \frac{(-1)^{s} q^{-\left(\frac{b}{a}+\frac{1}{2}\right) j s}}{[j-s-1]_{q} ![s]_{q} !}, \\
& =\frac{(-1)^{b / a j} q^{\left(\frac{b}{a}+\frac{1}{2}\right)\left(\begin{array}{l}
j \\
2
\end{array}\right)}}{j[j]_{q} !} \sum_{s=0}^{j-1}\left[\begin{array}{c}
j-1 \\
s
\end{array}\right]_{q}\left(-q^{b j / a}\right)^{s} q^{-\frac{j s}{2}}, \\
& =\frac{(-1)^{b / a j} q^{\left(\frac{b}{a}+\frac{1}{2}\right)\left(\begin{array}{l}
j \\
2
\end{array}\right)}}{j[j]_{q} !} \prod_{k=1}^{j-1}\left(1-q^{-k-b j / a}\right),
\end{aligned}
$$




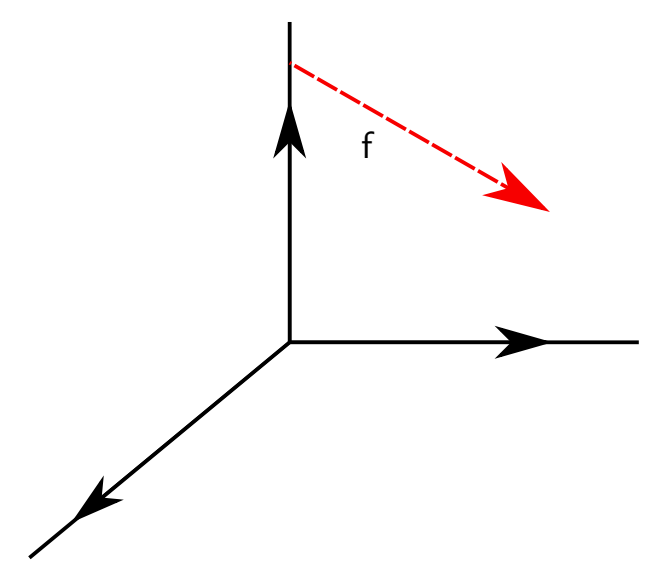

Figure 3.3. The toric graph of $(\mathbb{P}(1, a, b))^{\mathrm{op}}$, depicted for $a=3, b=2$.

$$
=\frac{(-1)^{b / a j}}{j[j]_{q} !} \frac{[(1+b / a) j-1]_{q} !}{[b / a j]_{q} !}=\frac{(-1)^{j b / a}}{j[(1+b / a) j]_{q}}\left[\begin{array}{c}
(1+b / a) j \\
j
\end{array}\right]_{q} .
$$

In this case, the map $\iota: \mathrm{H}_{1}(L, \mathbb{Z}) \rightarrow \mathrm{A}_{1}(\mathbb{P}(1, a, b))$ of 2.17$)$ is

$$
\iota:\left[S^{1}\right] \mapsto\left[D_{(-1,0)}\right]=\frac{H}{a},
$$

therefore, setting $j=d a$,

$$
\mathrm{O}_{d a}\left((\mathbb{P}(1, a, b))^{\mathrm{op}}\right)=\frac{(-1)^{d b}}{d a[(a+b) d]_{q}}\left[\begin{array}{c}
(a+b) d \\
a d
\end{array}\right]_{q},
$$

which proves Theorem 2.2 for $\mathbb{P}(1, a, b)$.

\section{EXAMPLE II}

Define $Y_{(a, b)}^{[2]}$ by considering the blow up $\pi: Y_{(a, b)}^{[2]} \longrightarrow \mathbb{P}(1, a, b)$ at a smooth point of the second divisor of $\mathbb{P}(1, a, b)$. Torically, we take the fan with rays $(-1,0),(-1,-1)(0,-1)$ and $(b, a)$ and choose $D_{1}=D_{(b, a)}$ as well as $D_{2}$ a smooth member of the linear system $D_{(-1,0)}+D_{(-1,-1)}+D_{(0,-1)}$. The relations are

$$
D_{(-1,0)}+D_{(-1,-1)} \sim b D_{1}, \quad D_{(0,-1)}+D_{(-1,-1)} \sim a D_{1},
$$

the intersections are

$$
D_{(-1,0)} \cdot D_{(-1,-1)}=D_{(-1,-1)} \cdot D_{(0,-1)}=1, \quad D_{(0,-1)} \cdot D_{(b, a)}=\frac{1}{b}, \quad D_{(-1,0)} \cdot D_{(b, a)}=\frac{1}{a},
$$

and the self-intersections are

$$
D_{(-1,0)}^{2}=\frac{b}{a}-1, \quad D_{(-1,-1)}^{2}=-1, \quad D_{(0,-1)}^{2}=\frac{a}{b}-1, \quad D_{(b, a)}^{2}=\frac{1}{a b} .
$$

Writing $D=D_{1}+D_{2}$, the topological Euler characteristic of the complement of $D$ is $\chi\left(Y_{(a, b)}^{[2]} \backslash D\right)=$ 2. Then, $\mathrm{H}_{2}\left(Y_{(a, b)}, \mathbb{Z}\right)$ is generated by the proper transform $\pi^{*} H \sim a b D_{1}$ and by the class of $D_{(-1,-1)}$. We write an effective curve class as $d=d_{0} f+d_{1} E$ with

$$
E:=D_{(-1,-1)}, \quad f:=\pi^{*} H-D_{(-1,-1)},
$$


so that

$$
d \cdot D_{1}=d_{0}, \quad d \cdot D_{2}=(a+b-1) d_{0}+d_{1}, \quad d \cdot \pi^{*} D_{(-1,0)}=b d_{0} \quad d \cdot D_{(-1,-1)}=d_{0}-d_{1} .
$$

4.1. Local GW invariants. In this case, the (inverse) Gram matrix of the $T$-equivariant Poincaré pairing in the basis $\left\{\varphi_{0}, \varphi_{1}, \varphi_{2}, \varphi_{3}\right\}=\{1, E, f$,pt $\}$ reads

$$
\eta_{Y_{(a, b)}^{[2]}}^{-1}=\left(\begin{array}{cccc}
0 & 0 & 0 & \lambda_{1} \lambda_{2} \\
0 & -\frac{\lambda_{1} \lambda_{2}(a b-1)}{a b} & \frac{\lambda_{1} \lambda_{2}}{a b} & -\frac{-a(b-1) \lambda_{1}+b \lambda_{1}+\lambda_{2}}{a b} \\
0 & \frac{\lambda_{1} \lambda_{2}}{a b} & \frac{\lambda_{1} \lambda_{2}}{a b} & -\frac{a \lambda_{1}+b \lambda_{1}+\lambda_{2}}{a b} \\
\lambda_{1} \lambda_{2} & -\frac{-a(b-1) \lambda_{1}+b \lambda_{1}+\lambda_{2}}{a b} & -\frac{a \lambda_{1}+b \lambda_{1}+\lambda_{2}}{a b} & \frac{1}{a}+\frac{1}{b}
\end{array}\right),
$$

and the $T$-equivariant $I$-function is

$$
\begin{aligned}
I_{(a, b)}^{Y^{[2]}}(y, z)= & \sum_{d_{0}, d_{1} \geq 0}\left[\frac{z y_{0}^{p_{0} / z+p_{1} / z} y_{0}^{d_{0}} y_{1}^{d_{1}} \prod_{m=0}^{d_{0}-1}\left(\lambda_{1}-p_{0}-m z\right)}{\prod_{m=1}^{d_{0}}\left(p_{0}+m z\right) \prod_{m=1}^{(b-1) d_{0}+d_{1}}\left((b-1) p_{0}+p_{1}+m z\right)}\right. \\
& \left.\frac{\prod_{m=0}^{(a+b-1) d_{0}+d_{1}-1}\left(\lambda_{2}+(1-b-a) p_{0}-p_{1}-m z\right)}{\prod_{m=1}^{d_{0}-d_{1}}\left(p_{0}-p_{1}+m z\right) \prod_{m=1}^{(a-1) d_{0}+d_{1}}\left((a-1) p_{0}+p_{1}+m z\right)}\right],
\end{aligned}
$$

where $p_{0}:=H /(a b), p_{1}:=H /(a b)-E$. At $\mathcal{O}(1)$ around $z=\infty$ we have

$$
I^{Y_{(a, b)}^{[2]}}(y, z)=z+\left(\log y_{0}\right) p_{0}+\left(\log y_{1}\right) p_{1}+\mathcal{O}\left(\frac{1}{z}\right)
$$

so the mirror map is once again trivial:

$$
J_{\text {small }}^{Y_{(a, b)}^{[2]}}(t, z)=I_{(a, b)}^{[2]}\left(e^{t}, z\right) .
$$

From (2.27) we obtain

$$
N_{d}^{\mathrm{loc}}\left(Y_{(a, b)}^{[2]}\right)=\sum_{i \in\{0,1,2,3\}}\left(\eta_{Y_{(a, b)}^{[2]}}\right)_{3, i}\left[\varphi_{i} z^{-1} y^{d}\right] I_{Y_{(a, b)}^{[2]}}(y, z)
$$

where, from (4.7), we have

$$
\left[z^{-1} y^{d}\right] I_{(a, b)}^{Y_{(2]}}(y, z)=\frac{\Gamma\left(d_{0}\right)\left(p_{0}-\lambda_{1}\right)(-1)^{d_{0}(a+b)+d_{1}} \Gamma\left(a d_{0}+b d_{0}-d_{0}+d_{1}\right)\left(p_{0}(a+b-1)-\lambda_{2}+p_{1}\right)}{\Gamma\left(d_{0}+1\right) \Gamma\left(d_{0}-d_{1}+1\right) \Gamma\left(a d_{0}-d_{0}+d_{1}+1\right) \Gamma\left(b d_{0}-d_{0}+d_{1}+1\right)} .
$$

Piecing 4.6), 4.10) and 4.11) together finally leads to

$$
N_{d}^{\mathrm{loc}}\left(Y_{(a, b)}^{[2]}\right)=\frac{(-1)^{d_{0}(a+b)+d_{1}}\left((a+b-1) d_{0}+d_{1}-1\right) !}{d_{0}\left(d_{0}-d_{1}\right) !\left((a-1) d_{0}+d_{1}\right) !\left((b-1) d_{0}+d_{1}\right) !} .
$$

\subsection{Log GW invariants.}

Proposition 4.1. Let $a, b$ be coprime positive integers. Then

$$
\mathrm{N}_{d}^{\log }\left(Y_{(a, b)}^{[2]}\right)(q)=\left[\begin{array}{c}
a d_{0} \\
d_{0}-d_{1}
\end{array}\right]_{q}\left[\begin{array}{c}
(a+b-1) d_{0}+d_{1} \\
a d_{0}
\end{array}\right]_{q}
$$

In conjunction with 4.12 , taking the $q \rightarrow 1$ limit recovers the numerical log-local correspondence of Theorem 2.1, 
Proof. Given that $Y_{(a, b)}$ differs from $\mathbb{P}(1, a, b)$ by blowing up a smooth point of $D_{2}$, the toric model of $Y_{(a, b)}^{[2]}$ differs from that of $\mathbb{P}(1, a, b)$ by additionally blowing up a smooth point of $\overline{D_{2}}$. At the level of scattering diagrams, we start with the one for $\mathbb{P}(1, a, b)$ as in Figure 3.2 and add a focusfocus singularity in the direction $(0,-1)$ (corresponding to $\overline{D_{2}}$ ). This creates simple scattering (see 15. Section 4.2]) as described in Figure 4.1. The broken line calculation proceeds as for $\mathbb{P}(1, a, b)$, with now both broken lines crossing a wall. Which walls which broken lines cross depends on the location of $p$. However, the end-result of multiplying the theta functions is independent from the location of $p$, see [41]. Figure 4.1 shows where the broken lines bend. As for $\mathbb{P}(1, a, b)$, the bending is determined by the intersection profile of $d$ with $D_{1}, D_{2}$ and the two exceptional divisors corresponding to the focus-focus singularities.

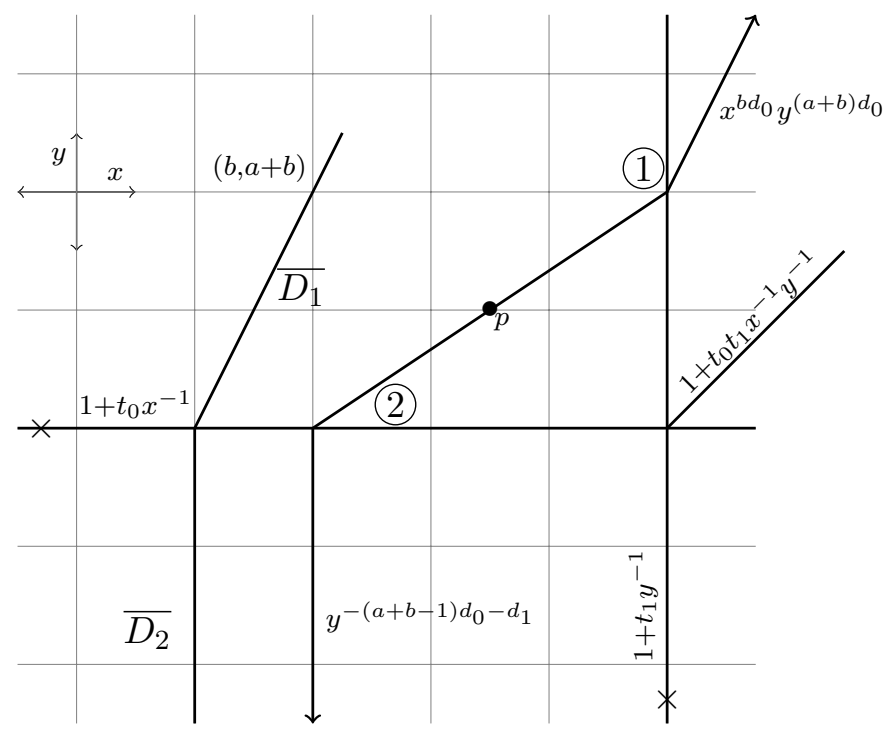

Figure 4.1. Scatt $Y_{(a, b)}^{[2]}$

At (1), the broken line coming from the $D_{1}$-direction picks up

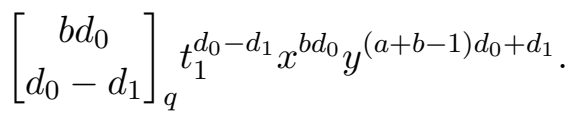

At (2), the broken line coming from the $D_{2}$-direction picks up

$$
\left[\begin{array}{c}
(a+b-1) d_{0}+d_{1} \\
b d_{0}
\end{array}\right]_{q} t_{0}^{b d_{0}} x^{-b d_{0}} y^{-(a+b-1) d_{0}-d_{1}} .
$$

We conclude by [15, Proposition 4.2], noting that

$$
\left[\begin{array}{c}
b d_{0} \\
d_{0}-d_{1}
\end{array}\right]_{q}\left[\begin{array}{c}
(a+b-1) d_{0}+d_{1} \\
b d_{0}
\end{array}\right]_{q}=\left[\begin{array}{c}
a d_{0} \\
d_{0}-d_{1}
\end{array}\right]_{q}\left[\begin{array}{c}
(a+b-1) d_{0}+d_{1} \\
a d_{0}
\end{array}\right]_{q} .
$$




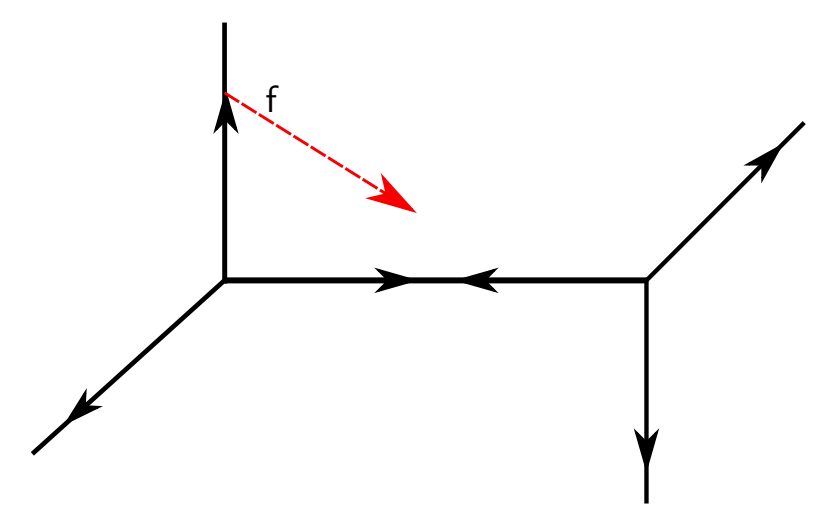

Figure 4.2. The toric graph of $\left(Y_{(a, b)}^{[2]}\right)^{\text {op }}$, depicted for $a=3, b=5$.

4.3. Open GW invariants. To construct $\left(Y_{(a, b)}^{[2]}\right)^{\text {op }}$, as for $\mathbb{P}(1, a, b)$, we take the canonical bundle on the complement of $D_{1}=D_{(b, a)}$. From the toric description of $Y_{(a, b)}^{[2]}$ we have that $Y_{(b, a)}^{[2]} \backslash D_{(b, a)}$ is described by a fan with 1-dimensional cones $D_{(-1,0)}, D_{(0,-1)}$ and $D_{(-1,-1)}$, hence $Y_{(b, a)}^{[2]} \backslash D_{(b, a)} \simeq$ $\mathcal{O}_{\mathbb{P}^{1}}(-1)$, and $K_{Y_{(b, a)}^{[2]} \backslash D_{(b, a)}} \simeq \mathcal{O}_{\mathbb{P}^{1}}(-1) \oplus \mathcal{O}_{\mathbb{P}^{1}}(-1)$. By [15. Construction 6.4], the compactification to $K_{Y_{(a, b)}^{[2]}}$ induces a framing of $b / a-1$ on the outer Lagrangian corresponding to the deletion of $D_{(b, a)}$; see Figure 4.2 .

From 2.37) and 2.38, we have

$$
\begin{aligned}
\mathcal{W}_{\alpha}\left(\left(Y_{(a, b)}^{[2]}\right)^{\mathrm{op}}\right)(Q, \hbar) & =q^{\left(\frac{b}{a}-1\right) \kappa(\alpha) / 2}(-1)^{\left(\frac{b}{a}-1\right)|\alpha|} \sum_{\nu \in \mathcal{P}} s_{\nu^{t}}\left(q^{\rho+\alpha}\right) s_{\alpha}\left(q^{\rho}\right) s_{\nu}\left(-Q q^{\rho}\right) \\
& =q^{\left(\frac{b}{a}-1\right) \kappa(\alpha) / 2}(-1)^{\left(\frac{b}{a}-1\right)|\alpha|} s_{\alpha}\left(q^{\rho}\right) \prod_{i, j \geq 1}\left(1-Q q^{-i-j+1+\alpha_{i}}\right),
\end{aligned}
$$

so that

$$
\frac{\mathcal{W}_{\left(j-s-1,1^{s}\right)}\left(\left(Y_{(a, b)}^{[2]}\right)^{\mathrm{op}}\right)(Q, \hbar)}{\mathcal{W}_{\emptyset}\left(\left(Y_{(a, b)}^{[2]}\right) \mathrm{op}\right)(Q, \hbar)}=\frac{\left.(-1)^{(b / a-1) j} q^{\left(\frac{b}{a}-\frac{1}{2}\right)}\right)\left(\left(\begin{array}{c}
j \\
2
\end{array}\right)-j s\right)}{\prod_{k=0}^{j-1}\left(1-q^{k} Q q^{-s}\right)} .
$$

Using the Cauchy binomial theorem we get

$$
\begin{aligned}
& \mathcal{O}_{j}\left(\left(Y_{(a, b)}^{[2]}\right)^{\mathrm{op}}\right)(Q, \hbar)=\sum_{s=0}^{j-1} \frac{(-1)^{s}}{j} \frac{\mathcal{W}_{\left(j-s-1,1^{s}\right)}\left(\left(Y_{(a, b)}^{[2]}\right)^{\mathrm{op}}\right)(Q, \hbar)}{\mathcal{W}_{\emptyset}\left(\left(Y_{(a, b)}^{[2]}\right) \mathrm{op}\right)(Q, \hbar)} \\
= & \frac{(-1)^{(b / a-1) j} q^{\left(\frac{b}{a}-\frac{1}{2}\right)\left(\begin{array}{l}
j \\
2
\end{array}\right)}}{j[j]_{q} !} \sum_{l=0}^{\infty} q^{\frac{l(j+1)}{2}}\left[\begin{array}{l}
j \\
l
\end{array}\right]_{q}(-Q)^{l} q^{-l} \sum_{s=0}^{j-1}\left[\begin{array}{c}
j-1 \\
s
\end{array}\right]_{q}\left(-q^{-(b / a-1) j-l}\right)^{s} q^{-\frac{1}{2} j s}, \\
= & \frac{(-1)^{(b / a-1) j} q^{\left(\frac{b}{a}-\frac{1}{2}\right)\left(\begin{array}{l}
j \\
2
\end{array}\right)}}{j[j]_{q} !} \sum_{l=0}^{\infty} q^{\frac{l(j-1)}{2}}\left[\begin{array}{l}
j \\
l
\end{array}\right]_{q}(-Q)^{l} \prod_{k=1}^{j-1}\left(1-q^{-(b / a-1) j-l-k}\right),
\end{aligned}
$$

so the $\mathcal{O}\left(Q^{l}\right)$ coefficient reads

$$
\mathrm{O}_{l ; j}\left(\left(Y_{(a, b)}^{[2]}\right)^{\mathrm{op}}\right)(\hbar)=\frac{(-1)^{(b / a+1) j+l}}{j[b / a j+l]_{q}}\left[\begin{array}{c}
b / a j+l \\
j
\end{array}\right]_{q}\left[\begin{array}{l}
j \\
l
\end{array}\right]_{q} .
$$


The morphism $\iota: \mathrm{H}_{2}\left(\mathbb{P}^{1}, \mathbb{Z}\right) \oplus \mathrm{H}_{1}\left(S^{1}, \mathbb{Z}\right) \rightarrow \mathrm{A}_{1}\left(Y_{(a, b)}^{[2]}\right)$ in 2.17 reads

$$
\begin{aligned}
& \iota:\left[S^{1}\right] \quad \mapsto\left[D_{(-1,0)}\right]=\frac{H}{a}-E, \\
& \iota:\left[\mathbb{P}^{1}\right] \mapsto\left[D_{(-1,-1)}\right]=E,
\end{aligned}
$$

and, accordingly, the change-of-variables relating the curve degrees $\left(d_{0}, d_{1}\right)$ in $\mathrm{H}_{2}\left(Y_{(a, b)}, \mathbb{Z}\right)$ and the relative homology variables $(l ; j)$ in $\mathrm{H}_{2}^{\mathrm{rel}}\left(\left(Y_{(a, b)}\right)^{\mathrm{op}}, L, \mathbb{Z}\right)$ is

$$
\begin{gathered}
j \rightarrow d_{0} a, \\
l \rightarrow(a-1) d_{0}+d_{1},
\end{gathered}
$$

verifying Theorem 2.2 for $Y_{(a, b)}^{[2]}$.

\section{EXAMPLE III}

Start with the toric pair $\mathbb{P}(1, a, b)$ and blow up a smooth point of the toric divisor $D_{(-1,0)} \sim H / a$, see Figure 1.1. The result is a toric surface $Y_{(a, b)}^{[3]}$ with non-toric boundary $D=D_{1}+D_{2}+D_{3}$ determined as follows. Torically, the fan of $Y_{(a, b)}^{[3]}$ is given by the rays directed by $(b, a),(-1,0)$, $(-1,-1)$ and $(0,-1)$. The boundary $D$ consists of two toric divisors, $D_{1}$ corresponding to the ray $(b, a)$ and $D_{2}$ corresponding to the ray $(-1,0)$, as well as a non-toric divisor $D_{3}$ which is a smooth member of the linear system determined by the sum of the rays $(-1,-1)$ and $(0,-1)$. The topological Euler characteristic of the complement of $D$ is $\chi\left(Y_{(a, b)}^{[3]} \backslash D\right)=1$. As before, we write an effective curve class as $d=d_{0} f+d_{1} E$, where $f, E \in \mathrm{H}_{2}\left(Y_{(a, b)}^{[3]}, \mathbb{Z}\right)$ are defined in 4.4$)$ and

$$
d \cdot D_{1}=d_{0}, \quad d \cdot D_{2}=(b-1) d_{0}+d_{1}, \quad d \cdot D_{3}=a d_{0}, \quad d \cdot E=d_{0}-d_{1} .
$$

5.1. Local GW invariants. In this case, the (inverse) Gram matrix of the $T$-equivariant Poincaré pairing in the basis $\left\{\varphi_{0}, \varphi_{1}, \varphi_{2}, \varphi_{3}\right\}=\{1, E, f$, pt $\}$ reads

$$
\eta_{Y_{(a, b)}^{[3]}}^{-1}=\left(\begin{array}{cccc}
0 & 0 & 0 & \lambda_{1} \lambda_{2} \lambda_{3} \\
0 & -\frac{\lambda_{1} \lambda_{2} \lambda_{3}(a b-1)}{a b} & \frac{\lambda_{1} \lambda_{2} \lambda_{3}}{a b} & \frac{b \lambda_{1}\left(a \lambda_{3}-\lambda_{2}\right)-\lambda_{3}\left(a \lambda_{1}+\lambda_{2}\right)}{a b} \\
0 & \frac{\lambda_{1} \lambda_{2} \lambda_{3}}{a b} & \frac{\lambda_{1} \lambda_{2} \lambda_{3}}{a b} & -\frac{\lambda_{3}\left(a \lambda_{1}+\lambda_{2}\right)+b \lambda_{1} \lambda_{2}}{a b} \\
\lambda_{1} \lambda_{2} \lambda_{3} & \frac{b \lambda_{1}\left(a \lambda_{3}-\lambda_{2}\right)-\lambda_{3}\left(a \lambda_{1}+\lambda_{2}\right)}{a b} & -\frac{\lambda_{3}\left(a \lambda_{1}+\lambda_{2}\right)+b \lambda_{1} \lambda_{2}}{a b} & \frac{\lambda_{2}}{a}+\frac{\lambda_{3}}{b}+\lambda_{1}
\end{array}\right),
$$

and the $T$-equivariant $I$-function is

$$
\begin{aligned}
& I_{(a, b)}^{Y_{(3]}}(y, z)=\sum_{d_{0}, d_{1} \geq 0}\left[\frac{\prod_{m=0}^{d_{0}-1}\left(\lambda_{1}-p_{0}-m z\right) \prod_{m=0}^{(a-1) d_{0}+d_{1}-1}\left(\lambda_{2}+(1-a) p_{0}-p_{1}-m z\right)}{\prod_{m=1}^{d_{0}}\left(p_{0}+m z\right) \prod_{m=1}^{(a-1) d_{0}+d_{1}}\left((a-1) p_{0}+p_{1}+m z\right)}\right. \\
& \left.\frac{z y_{0}^{p_{0} / z+p_{1} / z} y_{0}^{d_{0}} y_{1}^{d_{1}} \prod_{m=0}^{b d_{0}-1}\left(\lambda_{3}-b p_{0}-m z\right)}{\prod_{m=1}^{d_{0}-d_{1}}\left(p_{0}-p_{1}+m z\right) \prod_{m=1}^{(b-1) d_{0}+d_{1}}\left((b-1) p_{0}+p_{1}+m z\right)}\right] \text {, }
\end{aligned}
$$

where $p_{0}:=H /(a b), p_{1}:=H /(a b)-E$. It is immediate to check that there are no non-trivial contributions to the mirror map,

$$
\left[z^{0}\right] I^{Y_{(a, b)}^{[3]}}(y, z)=\underset{22}{\left(\log y_{0}\right)} p_{0}+\left(\log y_{1}\right) p_{1},
$$


and so we obtain

$$
J_{\text {small }}^{Y_{(a, b)}^{[3]}}(t, z)=I^{Y_{(a, b)}^{[3]}}\left(\mathrm{e}^{t}, z\right) .
$$

For the $\mathcal{O}(1 / z)$ term in the expansion of the $J$-functions we have quantum corrections only when $d_{0}=0$ or $d_{1}=(1-a) d_{0}$ :

$$
\begin{aligned}
{\left[z^{-1}\right] I^{Y_{(a, b)}^{[3]}}(y, z) } & =\frac{\left((1-a b) \log ^{2} y_{1}+\log ^{2} y_{0}+2 \log y_{1} \log y_{0}\right)}{2 a b} \mathrm{pt} \\
& +\sum_{d>0} \frac{(-1)^{(b+1) d}\left(p_{0}-\lambda_{1}\right)(b d-1) !\left(b p_{0}-\lambda_{3}\right) y_{1}^{d-a d} y_{0}^{d}}{d z(a d) !((b-a) d) !} \\
& +\sum_{d>0} \frac{\left(p_{1}-p_{0}\right)\left((a-1) p_{0}-\lambda_{2}+p_{1}\right) y_{1}^{d}}{d^{2}} .
\end{aligned}
$$

Acting on (5.6) with $\theta_{0}\left((b-1) \theta_{0}+\theta_{1}\right)$, where $\theta_{i}:=y_{i} \partial_{y_{i}}$, annihilates the quantum corrections in the last two rows. This entails that the small quantum cohomology product of $p_{0}$ and $(b-1) p_{0}+p_{1}$ is equal to their classical cup product:

$$
p_{0} \star_{y}\left((b-1) p_{0}+p_{1}\right)=p_{0} \cup\left((b-1) p_{0}+p_{1}\right)=\frac{H^{2}}{a^{2} b}=\frac{\mathrm{pt}}{a} .
$$

At the next order in $\mathcal{O}(1 / z)$, we have

$$
\begin{aligned}
& {\left[z^{-2}\right] I_{(a, b)}^{Y_{(3]}}(y, z)=} \\
& \sum_{\left(d_{0}, d_{1}\right) \neq(0,0)} \frac{y_{0}^{d_{0}} y_{1}^{d_{1}}(-1)^{d_{0}(a+b)+d_{1}}\left(b d_{0}-1\right) !\left(p_{0}-\lambda_{1}\right)\left((a-1) p_{0}-\lambda_{2}+p_{1}\right)\left(b p_{0}-\lambda_{3}\right)}{d_{0}\left((a-1) d_{0}+d_{1}\right)\left(d_{0}-d_{1}\right) !\left((b-1) d_{0}+d_{1}\right) !}
\end{aligned}
$$

so that

$$
\begin{aligned}
N_{d}^{\mathrm{loc}, \psi}\left(Y_{(a, b)}^{[3]}\right) & =\eta_{Y_{(a, b)}^{[3]}}\left(\mathrm{pt},\left[z^{-2} y_{0}^{d_{0}} y_{1}^{d_{1}}\right] I_{(a, b)}^{Y_{(3]}^{[3]}}\right) \\
& =\frac{(-1)^{d_{0}(a+b)+d_{1}+1}\left(a d_{0}-1\right) !}{d_{0}\left((b-1) d_{0}+d_{1}\right)\left(d_{0}-d_{1}\right) !\left((a-1) d_{0}+d_{1}\right) !}
\end{aligned}
$$

and thus

$$
\begin{aligned}
N_{d}^{\mathrm{loc}}\left(Y_{(a, b)}^{[3]}\right) & =\eta_{Y_{(a, b)}^{[3]}}\left(\mathrm{pt},\left[z^{-2} y_{0}^{d_{0}} y_{1}^{d_{1}}\right] b \theta_{0}\left((a-1) \theta_{0}+\theta_{1}\right) I_{(a, b)}^{\left.Y_{(3]}^{[3]}\right)}\right) \\
& =a d_{0}\left((b-1) d_{0}+d_{1}\right) N_{d}^{\mathrm{loc}, \psi}\left(Y_{(a, b)}^{[3]}\right) .
\end{aligned}
$$

\subsection{Log GW invariants.}

Proposition 5.1. Denote by $N_{d}^{\log , \psi}\left(Y_{(a, b)}^{[3]}\right)$ the genus 0 log $G W$ invariant of $Y_{(a, b)}^{[3]}$ with a point class with psi insertion [15, (4.2)]. Then

$$
\begin{aligned}
N_{d}^{\log , \psi}\left(Y_{(a, b)}^{[3]}\right) & =\left(\begin{array}{c}
a d_{0} \\
d_{0}-d_{1}
\end{array}\right), \\
\mathrm{N}_{d}^{\log }\left(Y_{(a, b)}^{[3]}\right)(\hbar) & =\frac{\left[a d_{0}\left((b-1) d_{0}+d_{1}\right)\right]_{q}}{[1]_{q}}\left[\begin{array}{c}
a d_{0} \\
d_{0}-d_{1}
\end{array}\right]_{q} .
\end{aligned}
$$




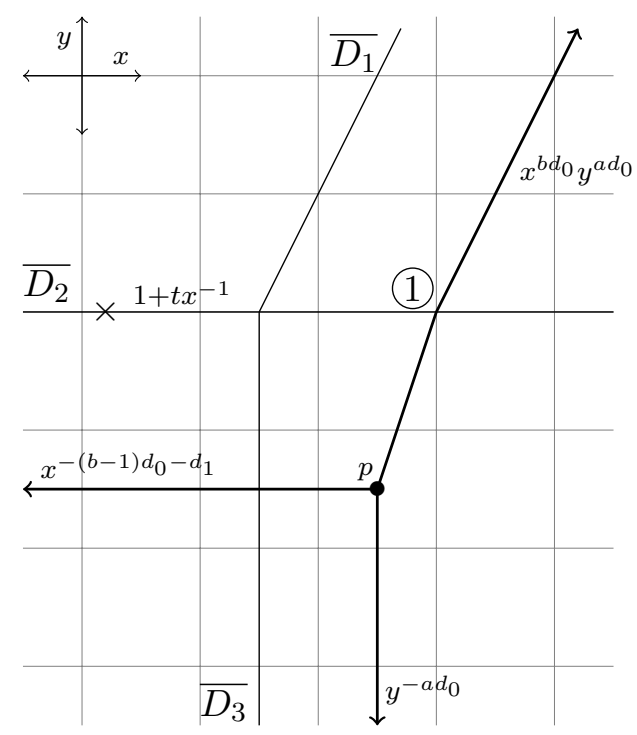

FiguRE 5.1. Scatt $Y_{(a, b)}^{[3]}$

The genus zero $(q \rightarrow 1)$ limit of Proposition 5.1, combined with (5.10), concludes the proof of Theorem 2.1.

Proof. Since $Y_{(a, b)}^{[3]}$ is obtained from $\mathbb{P}(1, a, b)$ by blowing up a smooth point on $D_{(-1,0)}$, the scattering diagram of $Y_{(a, b)}^{[3]}$ is given by the fan of $\mathbb{P}(1, a, b)$ with a focus-focus singularity in the direction $(-1,0)$. By [15, Section 4.2], we now need to extract the identity component of the multiplication of three broken lines corresponding to the directions $D_{i}$ with weights $d \cdot D_{i}, i=1,2,3$. The broken line calculation is given in Figure 5.1. Choosing the location of $p$ as in Figure 5.1, there is only one possible wall-crossing for the broken line coming from the $D_{1}$-direction. After crossing the wall at (1), the broken line coming from the $D_{1}$-direction carries the monomial

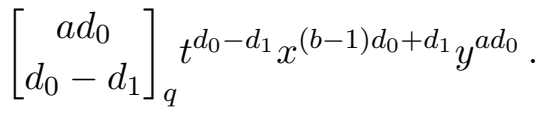

The result then follows from [15, Proposition 4.1] for the invariant with psi class and by 15 , Proposition 4.2] for the invariant with two point classes.

5.3. Open GW invariants. For $Y_{(a, b)}^{[3]}$ the relevant open geometry is a toric Lagrangian triple given by affine space $\mathbb{C}^{3}$ with two toric Lagrangians $L_{1}, L_{2}$ at framing $f_{1}=(a / b-1)$ and $f_{2}=0$; see Figure $5.2{ }^{3}$ For this setup, denoting $j_{i}$ the winding number of open stable maps around $S^{1} \hookrightarrow L_{i}, i=1,2$, we have

$$
\mathrm{O}_{j_{1}, j_{2}}\left(\left(Y_{(a, b)}^{[3]}\right)^{\mathrm{op}}\right)=\sum_{i_{1}=0}^{j_{1}-1} \sum_{i_{2}=0}^{j_{2}-1} \frac{(-1)^{i_{1}+i_{2}}}{j_{1} j_{2}} \mathcal{W}_{\left(j_{1}-i_{1}, 1^{i_{1}}\right),\left(j_{2}-i_{2}, 1^{\left.i_{2}\right)}\right.}^{(c)}\left(\left(Y_{(a, b)}^{[3]}\right)^{\mathrm{op}}\right),
$$

\footnotetext{
${ }^{3}$ A strict application of 15 . Construction 6.4] would in fact return $\mathbb{C}^{3}$ with two toric Lagrangians at framing $(-1,-a / b)$; the equivalence of these two open Gromov-Witten setups is a consequence of the well-known symmetries of the topological vertex, see 6. Section 3.4], as can also be easily verified in the foregoing formulas.
} 


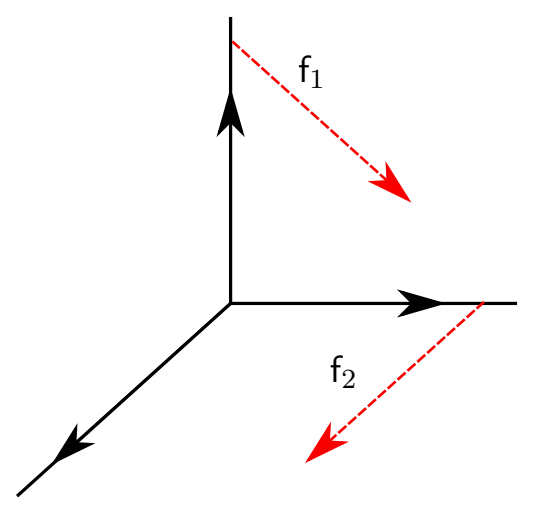

Figure 5.2. The toric graph of $\left(Y_{(a, b)}^{[3]}\right)$ op for $a=2, b=1$.

where $\mathcal{W}_{\alpha \beta}^{(c)}$ is the connected 2-leg topological vertex at framing $(a / b-1,0)$,

$$
\begin{aligned}
\mathcal{W}_{\alpha \beta}^{(c)}\left(\left(Y_{(a, b)}^{[3]}\right)^{\mathrm{op}}\right) & :=\mathcal{W}_{\alpha \beta}\left(\left(Y_{(a, b)}^{[3]}\right)^{\mathrm{op}}\right)-\mathcal{W}_{\alpha \emptyset}\left(\left(Y_{(a, b)}^{[3]}\right)^{\mathrm{op}}\right) \mathcal{W}_{\emptyset \beta}\left(\left(Y_{(a, b)}^{[3]}\right)^{\mathrm{op}}\right) \\
& =q^{a / b \kappa(\alpha) / 2}(-1)^{(a / b-1)|\alpha|} \sum_{\delta \neq \emptyset} s_{\frac{\alpha t}{\delta}}\left(q^{\rho}\right) s_{\frac{\beta}{\delta}}\left(q^{\rho}\right) .
\end{aligned}
$$

We can express (5.13) as a $q$-series using the $q$-factorial expression for hook skew Schur functions [15. App. C],

$$
s_{\frac{\left(d, 1^{i}\right)}{\delta}}\left(q^{\rho}\right)=\left\{\begin{array}{cc}
\frac{\left.(-1)^{-d-i+k+r} q^{\frac{1}{2}\left(d^{2}-2 d k+i+k^{2}-r\right.}\right)}{(q ; q)_{d-k}(q ; q)_{i-r}}, & \delta=\left(k, 1^{r}\right) \\
0 & \text { else }
\end{array}\right.
$$

as

$$
\mathrm{O}_{j_{1}, j_{2}}\left(\left(Y_{(a, b)}^{[3]}\right)^{\mathrm{op}}\right)=\frac{(-1)^{a / b j_{1}+j_{2}+1} q^{\frac{1}{2}\left(\frac{a j_{1}^{2}}{b}+j_{1}\left(\frac{a}{b}+1\right)+j_{2}^{2}\right)}}{j_{1} j_{2}} \sum_{k, l_{1}, l_{2}=0}^{\infty} \sum_{r=0}^{k-1} q^{\frac{a r j_{1}}{b}} \mathrm{a}_{l_{1}, j_{1}, k} \mathrm{~b}_{l_{2}, j_{2}, k} \mathrm{c}_{k},
$$

where we have shifted the indices of summation as $i_{1}=l_{1}+k-r-1, i_{2}=l_{2}+r$, and moreover

$$
\begin{aligned}
\mathrm{a}_{l_{1}, j_{1}, k} & :=\frac{(-1)^{l_{1}} q^{\frac{1}{2} l_{1}\left(l_{1}-1-2 a / b j_{1}\right)}}{(q ; q)_{l_{1}}(q ; q)_{j_{1}-k-l_{1}}}, \\
\mathrm{~b}_{l_{2}, j_{2}, k} & :=\frac{(-1)^{l_{2}} q^{\frac{1}{2} l_{2}\left(l_{2}+1+2 k-2 j_{2}\right)}}{(q ; q)_{l_{2}}(q ; q)_{j_{2}-k-l_{2}}} \\
\mathrm{c}_{k} & :=(-1)^{k} q^{\frac{1}{2} k\left(k-1-2 a / b j_{1}-2 j_{2}\right)} .
\end{aligned}
$$

In the formulas above, $(q ; q)_{n}$ is the usual $q$-Pochhammer symbol, $(q ; q)_{n}:=(-1)^{n} q^{\frac{1}{4} n(n+1)}[n]_{q} !$. Performing the $l_{2}$ summation using the Cauchy binomial theorem in the form

$$
(q y ; q)_{n}=\sum_{m=0}^{\infty} \frac{(-y)^{m} q^{\frac{m(m+1)}{2}}(q ; q)_{n}}{(q ; q)_{m}(q ; q)_{n-m}}
$$


gives

$$
\sum_{l_{2}=0}^{\infty} \mathrm{b}_{l_{2}, j_{2}, k}=\frac{\left(q^{k-j_{2}+1} ; q\right)_{j_{2}-k}}{(q ; q)_{j_{2}-k}}=\frac{1}{(q ; q)_{j_{2}-k}(q ; q)_{k-j_{2}}}=\delta_{j_{2} k}
$$

The sum over $k$ consists then of a single summand at $k=j_{2}$, and the sum over $l_{1}$, using (5.18), is

$$
\sum_{l_{1}=0}^{j_{1}-j_{2}} \mathrm{a}_{l_{1}, j_{1}, j_{2}}=(-1)^{j_{1}-j_{2}} q^{\frac{1}{2}\left(j_{2}-j_{1}\right)\left(j_{1} a / b+1\right)}\left[\begin{array}{c}
a j_{1} / b \\
j_{1}-j_{2}
\end{array}\right]_{q}
$$

Therefore,

$$
\begin{aligned}
\mathrm{O}_{j_{1}, j_{2}}\left(\left(Y_{(a, b)}^{[3]}\right)^{\mathrm{op}}\right) & =\frac{(-1)^{j_{1}(1+a / b)+1+j_{2}}}{j_{1} j_{2}} q^{-\frac{a j_{1}\left(j_{2}-1\right)}{2 b}}\left[\begin{array}{c}
a j_{1} / b \\
j_{1}-j_{2}
\end{array}\right]_{q} \sum_{r=0}^{j_{2}-1} q^{r j_{1} a / b} \\
& =(-1)^{j_{1}(1+a / b)+j_{2}+1}\left[\begin{array}{c}
a j_{1} / b \\
j_{1}-j_{2}
\end{array}\right]_{q} \frac{\left[j_{1} j_{2} a / b\right]_{q}}{\left[j_{1} a / b\right]_{q} j_{1} j_{2}} .
\end{aligned}
$$

From 2.17), the winding number variables in $\left(Y_{(a, b)}^{[3]}\right)^{\text {op }}$ and curve degrees in $Y_{(a, b)}^{[3]}$ are related as

$$
\begin{aligned}
& j_{1} \rightarrow b d_{0}, \\
& j_{2} \rightarrow(b-1) d_{0}+d_{1},
\end{aligned}
$$

which combined with (5.21) and Proposition 5.1 concludes the proof of Theorem 2.2 .

\section{BPS INVARIANTS}

The closed-form higher genus GW expressions of the previous Sections put us now in a position to prove Theorem 2.3. Write

$$
\Omega_{d}(Y(D))(q)=\frac{[1]_{q}^{2}}{\prod_{i=1}^{l}\left[d \cdot D_{i}\right]_{q}} \sum_{k \mid d}(-1)^{\sum_{i=1}^{l} d / k \cdot D_{i}+1} \mu(k)\left(\frac{[k]_{q}}{k}\right)^{3-l} \mathrm{~N}_{d / k}^{\log }(-\mathrm{i} k \log q) .
$$

From (3.1), 4.13) and (5.12), we have that obviously $\mathrm{N}_{d / k}^{\log }(-\mathrm{i} k \log q) \in \mathbb{Z}\left[q^{ \pm 1 / 2}\right]$ since it is a product of $q$-binomial coefficients. Then 6.1 implies a priori that $\Omega_{d}(Y(D))(q) \in \mathbb{Q}\left(q^{1 / 2}\right)$ with poles at $q=0, \infty$ and at most double poles at $q=\exp (2 \pi \mathrm{i} l / \hat{d})$, with $\hat{d}:=\operatorname{lcm}\left\{d \cdot D_{i}\right\}_{i=1}^{l}$. We have the following

Proposition 6.1. Let $Y(D)=\mathbb{P}(1, a, b), Y_{(a, b)}^{[2]}, Y_{(a, b)}^{[3]}$. Then

$$
\frac{\prod_{i=1}^{l}\left[d \cdot D_{i}\right]_{q}}{[1]_{q}^{2}} \Omega_{d}(Y(D))(q)=\mathcal{O}\left(q-\mathrm{e}^{\frac{2 \pi \mathrm{i} l}{d}}\right)^{2}, \quad l=0, \ldots, \hat{d}-1 .
$$

Proof. The vanishing at linear order can be shown with the exact same arguments of the proof of 15. Thm 8.1] by replacing therein $\Theta_{d}(q) \rightarrow \mathrm{N}_{d}^{\operatorname{loc}}(-\mathrm{i} \log q)\left(\right.$ for $\mathbb{P}(1, a, b)$ and $\left.Y_{(a, b)}^{[2]}\right)$ and $\Xi_{d}(q) \rightarrow$ $\mathrm{N}_{d}^{\operatorname{loc}}(-\mathrm{i} \log q)$ (for $\left.Y_{(a, b)}^{[3]}\right)$ : the summands in the divisor sums in 6.1 can be grouped in pairs with leading order terms at $q=\mathrm{e}^{\frac{2 \pi i l}{d}}$ having opposite signs, ensuring the l.h.s. is zero at that order. The quadratic vanishing is a consequence of [15, Lemma 8.3]. 


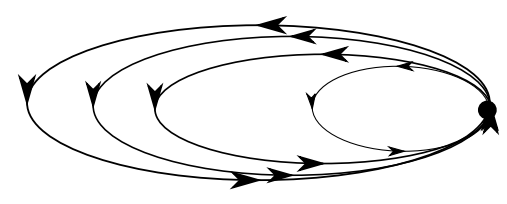

Figure 6.1. The quiver for $\mathbb{P}(1, a, b)$ for $a=1, b=3$.

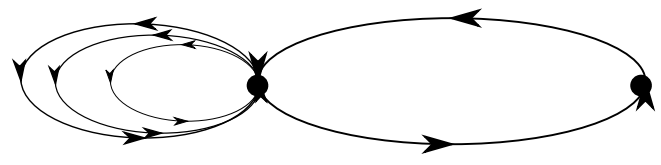

Figure 6.2. The quiver for $Y_{(a, b)}^{[2]}$ for $a=1, b=3$.

The Proposition then implies that $\Omega_{d}(Y(D)) \in \mathbb{Q}\left[q^{ \pm 1 / 2}\right]$. Since $1 /\left[d \cdot D_{i}\right]_{q} \in q^{-d \cdot D_{i} / 2} \mathbb{Z}[[q]]$, from 6.1 ) we have $\Omega_{d}(Y(D)) \in \mathbb{Z}\left[q^{-1 / 2}\right]\left[\left[q^{1 / 2}\right]\right]$, and thus $\Omega_{d}(Y(D)) \in \mathbb{Z}\left[q^{ \pm 1 / 2}\right]$ from the previous Proposition. The claim of Theorem 2.3 then follows.

6.1. Quiver DT invariants. For $l=2$ and whenever $a=1$ or $b=1, Y^{\mathrm{op}}(D)$ is an integrally framed toric Lagrangian triple of 'strip' type [57], that is, it consists of a single integrally framed outer Aganagic-Vafa Lagrangian on a smooth toric Calabi-Yau threefold whose fan is a cone over an integral polytope of unit width. Then Theorem 2.4 follows from a proof identical in all its parts to that of [15. Thm 7.3], with framings now equal to $f=b$ (for $\mathbb{P}(1, a, b)$ with $a=1)$ and $f=b-1$ (for $Y_{(a, b)}^{[2]}$ with $\left.a=1\right)$.

Example 6.1. For $Y(D)=\mathbb{P}(1,1, b)^{[2]}, Y^{\mathrm{op}}(D)$ is the 1-legged vertex at framing $b$, for which the corresponding quiver is the $b+1$-loop quiver 57] (see Figure 6.1). The dimension vector is here identically identified with the curve degree $d, \kappa=$ id, and the integral shift in Theorem 2.4 vanishes, $\alpha_{i}=0$. The Klemm-Pandharipande invariants $\mathrm{KP}_{d}\left(E_{\mathbb{P}(1,1, b)^{[2]}}\right)$ are then up to a sign the polynomials in $\frac{1}{d !} \mathbb{Z}[b]$ computed by Reineke in [58, Thm 3.2]. Explicitly, we have

$$
\begin{aligned}
\mathrm{KP}_{d}\left(E_{\left.\mathbb{P}(1,1, b)^{[2]}\right)=}\right. & \left\{(-1)^{b}, \frac{1}{4}\left((2 b+1)-(-1)^{b}\right), \frac{1}{2}(-1)^{b} b(b+1),\right. \\
& \left.\frac{1}{3} b(b+1)(2 b+1), \frac{5}{24}(-1)^{b} b(b+1)(5 b(b+1)+2), \ldots\right\} \\
= & (-1)^{b+d+1} \mathrm{DT}_{d}\left(\mathrm{Q}\left(\mathbb{P}(1,1, b)^{[2]}\right)\right)
\end{aligned}
$$

Example 6.2. For $Y(D)=Y_{(1, b)}^{[2]}$, the corresponding quiver is given in Figure 6.2

The map between vertices of the quiver and effective generators of $\mathrm{H}_{2}\left(Y_{(1, b)}, \mathbb{Z}\right)$ is

$$
\begin{array}{ll}
v_{1} \stackrel{\kappa}{\longrightarrow} & f \\
v_{2} & \stackrel{\kappa}{\longrightarrow} E
\end{array}
$$

and the integral shifts in Theorem 2.4 are $\alpha_{1}=0, \alpha_{2}=1$. Klemm-Pandharipande invariants for small degrees $d_{0}, d_{1}>0$ are given in Table 1: note that despite being rational polynomials in $b$, 
they take integer values for $b \in \mathbb{Z}^{+}$. The quiver DT invariants of $\mathrm{Q}\left(Y_{(1, b)}^{[2]}\right)$ are obtained as their absolute values.

\begin{tabular}{|c|c|c|c|c|}
\hline$d_{0}$ & 1 & 2 & 3 & 4 \\
\hline 1 & $(-1)^{b}$ & 0 & 0 & 0 \\
\hline 2 & $-b$ & $\frac{(-1)^{b}\left((-1)^{b}(2 b+1)-1\right)}{4}$ & 0 & 0 \\
\hline 3 & $\frac{(-1)^{b} b(3 b-1)}{2}$ & $-\frac{(-1)^{b} b(3 b+1)}{2}$ & $\frac{(-1)^{b} b(b+1)}{2}$ & 0 \\
\hline 4 & $-\frac{b\left(8 b^{2}-6 b+1\right)}{3}$ & $4 b^{3}$ & $-\frac{b(2 b+1)(4 b+1)}{3}$ & $\frac{b(b+1)(2 b+1)}{3}$ \\
\hline 5 & $\frac{(-1)^{b} b(5 b-3)(5 b-2)(5 b-1)}{24}$ & $\frac{(-1)^{b+1} b(5 b-2)(5 b-1)(5 b+1)}{12}$ & $\frac{(-1)^{b} b(5 b-1)(5 b+1)(5 b+2)}{12}$ & $\frac{(-1)^{b+1} b(5 b+1)(5 b+2)(5 b+3)}{24}$ \\
\hline
\end{tabular}

TABLE 1. BPS/KP invariants of $Y_{(1, b)}^{[2]}$.

Acknowledgements. Boris Dubrovin has been a figure of immense importance for the fields of Geometry and Mathematical Physics in general, and an attentive and kind mentor for some of us in particular. Even if centred in a topic far from the theory of integrable systems, this paper bears a very direct intellectual debt to Boris' work (most crucially, in the 2-point reconstruction lemma proving Theorem 2.1 for $Y_{(a, b)}^{[3]}$ ). It is a privilege to be able to dedicate this paper as a modest testament to his legacy.

\section{REFERENCES}

[1] D. Abramovich and Q. Chen, Stable logarithmic maps to Deligne-Faltings pairs II, Asian J. Math., 18 (2014), 465-488.

[2] D. Abramovich, T. Graber, and A. Vistoli, Gromov-Witten theory of Deligne-Mumford stacks, Amer. J. Math., 130 (2008), 1337-1398.

[3] D. Abramovich and J. Wise, Birational invariance in logarithmic Gromov-Witten theory, Compos. Math., 154 (2018), 595-620.

[4] M. Aganagic, R. Dijkgraaf, A. Klemm, M. Marino, and C. Vafa, Topological strings and integrable hierarchies, Commun. Math. Phys., 261 (2006), 451-516. Preprint available at hep-th/0312085.

[5] M. Aganagic, T. Ekholm, L. NG, and C. Vafa, Topological Strings, D-Model, and Knot Contact Homology, Adv. Theor. Math. Phys., 18 (2014), 827-956. Preprint available at 1304.5778.

[6] M. Aganagic, A. Klemm, M. Marino, and C. Vafa, The topological vertex, Commun. Math. Phys., 254 (2005), 425-478. Preprint available at hep-th/0305132.

[7] M. Aganagic and C. Vafa, Mirror symmetry, D-branes and counting holomorphic discs. Preprint available at hep-th/0012041.

[8] L. Battistella, N. Nabijou, H.-H. Tseng, and F. You, The local-orbifold correspondence for simple normal crossings pairs. Preprint available at 2103.09299.

[9] P. Bousseau, A proof of N. Takahashi's conjecture on genus zero Gromov-Witten theory of $\left(\mathbb{P}^{2}, E\right)$. Preprint available at 1909.02992 .

[10] Scattering diagrams, stability conditions, and coherent sheaves on $\mathbb{P}^{2}$. Preprint available at 1909.02985.

[11] _ Tropical refined curve counting from higher genera and lambda classes, Invent. Math., 215 (2019), 1-79.

[12] _ Quantum mirrors of log Calabi-Yau surfaces and higher-genus curve counting, Compos. Math., 156 (2020), 360-411.

[13] The quantum tropical vertex, Geom. Topol., 24 (2020), 1297-1379. 
[14] P. Bousseau, A. Brini, and M. van Garrel, On the log-local principle for the toric boundary. Preprint available at 1908.04371 .

[15] Stable maps to Looijenga pairs. Preprint available at 2011.08830.

[16] P. Bousseau, H. Fan, S. Guo, and L. Wu, Holomorphic anomaly equation for $\left(\mathbb{P}^{2}, E\right)$ and the NekrasovShatashvili limit of local $\mathbb{P}^{2}$, Forum Math. Pi, 9 (2021), e3, 57.

[17] A. Brini And R. CAvalieri, Open orbifold Gromov-Witten invariants of $\left[\mathbb{C}^{3} / \mathbb{Z}_{n}\right]$ : localization and mirror symmetry, Selecta Math. (N.S.), 17 (2011), 879-933. Preprint available at 1007.0934.

[18] A. Brini, B. Eynard, And M. Marino, Torus knots and mirror symmetry, Annales Henri Poincare, 13 (2012), 1873-1910. Preprint available at 1105.2012.

[19] Y. CaO, M. Kool, and S. Monavari, Stable pair invariants of local Calabi-Yau 4-folds. Preprint available at 2004.09355.

[20] Y. CaO, D. Maulik, And Y. Toda, Stable pairs and Gopakumar-Vafa type invariants for Calabi-Yau 4-folds. Preprint available at 1902.00003.

[21] Q. Chen, Stable logarithmic maps to Deligne-Faltings pairs I, Ann. of Math. (2), 180 (2014), $455-521$.

[22] D. Cheong, I. Ciocan-Fontanine, and B. Kim, Orbifold quasimap theory, Math. Ann., 363 (2015), $777-816$.

[23] J. Choi, M. van Garrel, S. KAtz, And N. TAKAhashi, Local BPS invariants: enumerative aspects and wall-crossing, Int. Math. Res. Not. IMRN, 17 (2020), 5450-5475.

[24] L L L L B BS numbers of log Calabi-Yau surfaces, Trans. Amer. Math. Soc., 374 (2021), 687-732.

[25] Sheaves of maximal intersection and multiplicities of stable log maps, Selecta Math. (N.S.), 27, 61 (2021). Preprint available at 1908.10906.

[26] T. Coates, A. Corti, H. Iritani, and H-H. Tseng, Computing genus-zero twisted Gromov-Witten invariants, Duke Math. J., 147 (2009), 377-438.

[27] _ A mirror theorem for toric stacks, Compos. Math., 151 (2015), 1878-1912.

[28] T. Coates and A. Givental, Quantum Riemann-Roch, Lefschetz and Serre, Ann. of Math. (2), 165 (2007), 15-53.

[29] B. Davison And T. MAndel, Strong positivity for quantum theta bases of quantum cluster algebras. Preprint available at 1910.12915 .

[30] D. E. Diaconescu, V. Shende, and C. VAfa, Large N duality, Lagrangian cycles, and algebraic knots, Commun. Math. Phys., 319 (2013), 813-863. Preprint available at 1111.6533.

[31] B. Dubrovin, Geometry of 2D topological field theories, in "Integrable systems and quantum groups" (Montecatini Terme, 1993), Lecture Notes in Math., 1620 (1994), 120-348. Preprint available at hep-th/9407018.

[32] A. I. Efimov, Cohomological Hall algebra of a symmetric quiver, Compos. Math., 148 (2012), $1133-1146$.

[33] T. Ekholm, P. Kucharski, And P. Longhi, Physics and geometry of knots-quivers correspondence, Commun. Math. Phys., 379 (2020), 361-415. Preprint available at 1811.03110.

[34] H. Fan, H.-H. Tseng, And F. You, Mirror theorems for root stacks and relative pairs, Selecta Math. (N.S.), 25 (2019), Paper No. 54, 25.

[35] B. Fang And Z. Zong, Topological recursion for the conifold transition of a torus knot, Selecta Math. (N.S.), 25 (2019), Paper No. 35, 44.

[36] M. van Garrel, T. Graber, and H. Ruddat, Local Gromov-Witten invariants are log invariants, Adv. Math., 350 (2019), 860-876.

[37] A. B. Givental, Equivariant Gromov-Witten invariants, Internat. Math. Res. Notices, 13 (1996), 613-663.

[38] _ A mirror theorem for toric complete intersections, Progr. Math., 160 (1998), 141-175.

[39] M. Gross, Tropical geometry and mirror symmetry. CBMS Regional Conference Series in Mathematics, 114. Published for the Conference Board of the Mathematical Sciences, Washington, DC; by the American Mathematical Society, Providence, RI, 2011.

[40] M. Gross, P. Hacking, And S. KeEl, Mirror symmetry for log Calabi-Yau surfaces I, Publ. Math. Inst. Hautes Études Sci., 122 (2015), 65-168. 
[41] M. Gross, P. Hacking, And B. Siebert, Theta functions on varieties with effective anti-canonical class, Mem. Amer. Math. Soc. (2020). Preprint available at 1601.07081.

[42] M. Gross, R. Pandharipande, and B. Siebert, The tropical vertex, Duke Math. J., 153 (2010), $297-362$.

[43] M. Gross And B. Siebert, From real affine geometry to complex geometry, Ann. of Math. (2), 174 (2011), 1301-1428.

[44] _ Logarithmic Gromov-Witten invariants, J. Amer. Math. Soc., 26 (2013), 451-510.

[45] E. N. Ionel And T. H. PARKer, The Gopakumar-Vafa formula for symplectic manifolds, Ann. of Math. (2), 187 (2018), 1-64.

[46] A. Klemm and R. Pandharipande, Enumerative geometry of Calabi-Yau 4-folds, Commun. Math. Phys., 281 (2008), 621-653. Preprint available at math/0702189.

[47] P. Kucharski, M. Reineke, M. Stosic, and P. Sulkowski, Knots-quivers correspondence, Adv. Theor. Math. Phys., 23 (2019), 1849-1902. Preprint available at 1707.04017.

[48] J. M. F. Labastida And M. Marino, Polynomial invariants for torus knots and topological strings, Commun. Math. Phys., 217 (2001), 423-449. Preprint available at hep-th/0004196.

[49] J. M. F. Labastida, M. Marino, And C. Vafa, Knots, links and branes at large N, JHEP, 11 (2000), 007. Preprint available at hep-th/0010102.

[50] J. Li, C-C. M. Liu, K. Liu, And J. Zhou, A mathematical theory of the topological vertex, Geom. Topol., 13 (2009), 527-621. Preprint available at math/0408426.

[51] T. MAndel, Scattering diagrams, theta functions, and refined tropical curve counts. Preprint available at 1503.06183.

[52] M. Marino And C. Vafa, Framed knots at large N, Contemp. Math., 310 (2002), 185-204. Preprint available at hep-th/0108064.

[53] D. Maulik, A. Oblomkov, A. Okounkov, and R. Pandharipande, Gromov-Witten/Donaldson-Thomas correspondence for toric 3-folds, Invent. Math. (2008), 1-45. Preprint available at arXiv:0809.3976.

[54] D. Maulik, R. Pandharipande, and R. P. Thomas, Curves on $K 3$ surfaces and modular forms, J. Topol., 3 (2010), 937-996.

[55] N. Nabijou And D. Ranganathan, Gromov-Witten theory with maximal contacts. Preprint available at 1908.04706.

[56] H. Ooguri And C. VAfa, Knot invariants and topological strings, Nucl. Phys., B577 (2000), 419-438. Preprint available at hep-th/9912123.

[57] M. Panfil, M. Stosic, and P. Sulkowski, Donaldson-Thomas invariants, torus knots, and lattice paths, Phys. Rev. D, 98 (2018), 026022. Preprint available at 1802.04573.

[58] M. Reineke, Degenerate cohomological Hall algebra and quantized Donaldson-Thomas invariants for $m$-loop quivers, Doc. Math., 17 (2012), 1-22.

[59] R. P. Stanley, Theory and application of plane partitions. I, II, Studies in Appl. Math., 50 (1971), 167-188; ibid. 50 (1971), 259-279.

[60] H-H. Tseng, Orbifold quantum Riemann-Roch, Lefschetz and Serre, Geom. Topol., 14 (2010), 1-81.

[61] H-H. Tseng And F. You, A mirror theorem for multi-root stacks and applications. Preprint available at 2006.08991.

[62] M. van Garrel, T. W. H. Wong, and Gu. Zaimi, Integrality of relative BPS state counts of toric del Pezzo surfaces, Commun. Number Theory Phys., 7 (2013), 671-687. 
Université Paris-Saclay, CNRS, Laboratoire de mathématiques d'Orsay, 91405, Orsay, France

Email address: pierrick.bousseau@u-psud.fr

School of Mathematics and Statistics, University of Sheffield, S11 9DW, Sheffield, United Kingdom. On leave from CnRS, DR 13, Montpellier, France

Email address: a.brini@sheffield.ac.uk

University of Birmingham, School of Mathematics, B15 2Tt, Birmingham, United Kingdom

Email address: m.vangarrel@bham.ac.uk 